\title{
Impurity spin textures across conventional and deconfined quantum critical points of two- dimensional antiferromagnets
}

\section{Citation}

Metlitski, Max A., and Subir Sachdev. 2007. "Impurity Spin Textures across Conventional and Deconfined Quantum Critical Points of Two-Dimensional Antiferromagnets." Physical Review B 76 (6). https://doi.org/10.1103/physrevb.76.064423.

\section{Permanent link}

http://nrs.harvard.edu/urn-3:HUL.InstRepos:41417247

\section{Terms of Use}

This article was downloaded from Harvard University's DASH repository, and is made available under the terms and conditions applicable to Other Posted Material, as set forth at http:// nrs.harvard.edu/urn-3:HUL.InstRepos:dash.current.terms-of-use\#LAA

\section{Share Your Story}

The Harvard community has made this article openly available. Please share how this access benefits you. Submit a story. 


\title{
Impurity spin textures across conventional and deconfined quantum critical points of two-dimensional antiferromagnets
}

\author{
Max A. Metlitski* and Subir Sachdev ${ }^{\dagger}$ \\ Department of Physics, Harvard University, Cambridge MA 02138, USA
}

(Dated: November 24, 2018)

\begin{abstract}
We describe the spin distribution in the vicinity of a non-magnetic impurity in a two-dimensional antiferromagnet undergoing a transition from a magnetically ordered Néel state to a paramagnet with a spin gap. The quantum critical ground state in a finite system has total spin $S=1 / 2$ (if the system without the impurity had an even number of $S=1 / 2$ spins), and recent numerical studies in a double layer antiferromagnet (K. H. Höglund et al., Phys. Rev. Lett. 98, 087203 (2007)) have shown that the spin has a universal spatial form delocalized across the entire sample. We present the field theory describing the uniform and staggered magnetizations in this spin texture for two classes of antiferromagnets: $(i)$ the transition from a Néel state to a paramagnet with local spin singlets, in models with an even number of $S=1 / 2$ spins per unit cell, which are described by a $\mathrm{O}$ (3) Landau-Ginzburg-Wilson field theory; and (ii) the transition from a Néel state to a valence bond solid, in antiferromagnets with a single $S=1 / 2$ spin per unit cell, which are described by a "deconfined" field theory of spinons.
\end{abstract}




\section{INTRODUCTION}

There have been many experimental studies of non-magnetic Zn impurities substituting for the spin $S=1 / 2 \mathrm{Cu}$ ions in spin-gap and superconducting compounds ${ }^{1-6}$. These have stimulated many theoretical studies of the spin dynamics in the vicinity of a vacancy (i.e. a site with no spin) in $S=1 / 2$ square lattice antiferromagnets ${ }^{7-19}$.

An important feature of the impurity-response escaped ${ }^{1}$ theoretical attention until recently ${ }^{18}$. Consider the regime where the bulk antiferromagnet preserves global rotational symmetry and has a $S=0$ ground state. Such states can be reached by deforming the nearest-neighbor antiferromagnet into a coupled-ladder or coupled-dimer antiferromagnet $^{20,21}$, in a double-layer antiferromagnet ${ }^{22}$, or by adding additional ringexchange interactions while preserving full square lattice symmetry ${ }^{23}$. Now remove a single $S=1 / 2$ spin in a system with an even number of spins, leaving an antiferromagnet with a vacancy and an odd number of $S=1 / 2$ spins. We expect this antiferromagnet to have a doubly-degenerate ground state with total spin $S=1 / 2$. Without loss of generality, we can examine the ground state with spin-projection $S_{z}=1 / 2$. In such a state, even though there is no broken symmetry and no applied magnetic field (the Hamiltonian has full SU(2) spin symmetry), the expectation values of the spin projection on the site $i,\left\langle S_{z i}\right\rangle$, is non-zero on all $i$ for any finite system of size $L$. The question of interest in this paper is the following: What is the spatial form of $\left\langle S_{z i}\right\rangle$ ? It is possible that the $S=1 / 2$ magnetization is pushed out to the boundaries of the system, far from the impurity: in this case, it will not be relevant to the impurity properties in the limit $L \rightarrow \infty$. However, we will find this is not the case for the antiferromagnets examined in this paper. For the spin-gap antiferromagnets we consider, the $S=1 / 2$ magnetization is bound to the impurity over a length scale inversely proportional to the spin gap. At the quantum critical points separating the spin gap states from the Néel state, which define 'algebraic spin liquids', we will find, as in Ref. 18, that the impurity magnetization is delocalized over the entire system, forming a spin texture with a universal spatial form determined only by the system size $L$.

First, let us consider the models which have been numerically studied in Ref. 18. These are antiferromagnets which have an even number of $S=1 / 2$ spins per unit cell (such as the coupled-dimer ${ }^{20,21}$ or double layer ${ }^{22}$ models), which exhibit a transition between a Néel state and a simple spin gap state; the latter state is adiabatically connected to a state in which the spins in each unit cell are separately locked into singlets, with negligible resonance between unit cells. This is a 'conventional' transition, described by a Landau-GinzburgWilson (LGW) theory. A convenient description of both phases and the quantum phase transition is provided by the $\mathrm{O}(3)$ non-linear sigma model, expressed in terms of a unit vector field $\mathbf{n}(\vec{x}, \tau)$ representing the local orientation of the Néel order parameter. Here $\vec{x}$ is the two-dimensional spatial position, $\tau$ is imaginary time, and $\mathbf{n}^{2}=1$ everywhere in spacetime. The bulk action in the absence of the impurity is the $\mathrm{O}(3)$ non-linear sigma

\footnotetext{
${ }^{1}$ Section II.B.3 of Ref. 15 contains results which can be used to extract the spin textures in zero field.
} 
model

$$
\mathcal{S}_{\mathrm{b}}^{\mathbf{n}}=\frac{1}{2 g} \int d \tau \int d^{2} x\left(\partial_{\mu} \mathbf{n}\right)^{2}
$$

where $g$ is the coupling constant which tunes the antiferromagnet from the Néel state $\left(g<g_{c}\right)$ to the spin gap state $\left(g>g_{c}\right), \mu$ is a 3-dimensional spacetime index and a spin-wave velocity has been set to unity. In this formulation, the influence of the impurity is represented universally by the following Berry phase term alone ${ }^{15}$ (provided the antiferromagnet is not too far from the critical point)

$$
\mathcal{S}_{\text {imp }}^{\mathbf{n}}=i S \int d \tau \mathbf{A}[\mathbf{n}(0, \tau)] \cdot \frac{d \mathbf{n}(0, \tau)}{d \tau}
$$

for a spin $S=1 / 2$ antiferromagnet, where $\mathbf{A}$ is the Dirac monopole function in spin space with $\nabla_{\mathbf{n}} \times \mathbf{A}=\mathbf{n}$. Note that $\mathcal{S}_{\mathrm{imp}}^{\mathbf{n}}$ does not include any coupling constants, and it depends upon the value of $\mathbf{n}$ only at $\vec{x}=0$, which is the position of the impurity.

Now we need to describe the $S=1 / 2$ ground state of $\mathcal{S}_{b}^{\mathbf{n}}+\mathcal{S}_{\text {imp }}^{\mathbf{n}}$ for $g \geq g_{c}$. First, we need a proper discussion of the rotationally invariant $S=0$ ground state without the impurity. While it may be possible to do this within the context of a small $g$ expansion of the $\mathrm{O}(3)$ non-linear sigma model, the procedure is quite cumbersome and delicate, requiring a global average over all possible locally ordered states. We shall instead follow a simpler procedure which is described in more detail in Section II: we use an alternative soft-spin, LGW formulation of $\mathcal{S}_{b}^{\mathbf{n}}$ in terms of a vector order parameter, $\phi$, whose length is unconstrained. The $\phi=0$ saddle point then is an appropriate starting point for describing the physics of the $S=0$ ground state of the bulk theory and its excitations. Next, we include the impurity term described by $\mathcal{S}_{\text {imp }}^{\mathbf{n}}$, and also apply an infinitesimal magnetic field in the $z$ direction. As we will show in Section II, the Berry phase effectively localizes the order parameter at the impurity site, $\mathbf{n}(\vec{x}=0, \tau)$, to a specific orientation on the unit sphere; in particular, for the $S_{z}=1 / 2$ state chosen by the applied field, we may perform an expansion about a saddle point with $\mathbf{n}(\vec{x}=0, \tau)=(1,0,0)$. This expansion quantizes, at each order, the total spin at $S_{z}=1 / 2$ : this was established in Section II.C.2 of Ref. 10 for $g<g_{c}$, and the same result also applies here for $g \geq g_{c}$. The infinitesimal magnetic field is set to zero at the end, but the spin density of the $S_{z}=1 / 2$ state remains non-zero in this limit.

The results in Section II provide an explicit analytic realization for the scaling forms presented in Ref. 18 for the spin texture near the impurity. For the magnetization density, Q, which is the conserved Noether "charge" density associated with the $\mathrm{O}(3)$ symmetry of the antiferromagnet, we have at $g=g_{c}$ and zero temperature $(T)$ and in the $S_{z}=1 / 2$ state:

$$
\left\langle Q_{z}(\vec{x})\right\rangle=\frac{1}{L^{2}} \Phi_{Q}\left(\frac{\vec{x}}{L}\right)
$$


where $\Phi_{Q}(\vec{r})$ is a universal function obeying the quantized total spin condition

$$
\int d^{2} r \Phi_{Q}(\vec{r})=S
$$

Similarly, the staggered magnetization associate with the Néel order parameter obeys the scaling form

$$
\left\langle n_{z}(\vec{x})\right\rangle=\frac{1}{L^{(1+\eta) / 2}} \Phi_{n}\left(\frac{\vec{x}}{L}\right)
$$

at $g=g_{c}$, where $\Phi_{n}(\vec{r})$ is another universal function, but its overall scale is non-universal. The exponent $\eta$ is the anomalous dimension of $\mathbf{n}$ at $g=g_{c}$ in the absence of the impurity.

Let us now turn to the more interesting and much more subtle case of a "deconfined" critical point ${ }^{24,25}$. Here we are considering antiferromagnets with a single $S=1 / 2$ spin per unit cell, and so there is no simple spin-gap state with local singlets. For the models studied in Refs. 23-25, the spin gap state has singlet valence bonds which crystallize into a regular arrangement, breaking the space group symmetry of the square lattice, while preserving spin rotation invariance. Such a state is a valence bond solid (VBS), and we are now interested in the impurity response across the Néel-VBS transition. As argued in Refs. 24,25 the vicinity of this quantum critical point is described by the $\mathbb{C P}^{1}$ field theory which is expressed in terms of a bosonic 'spinon' represented by a complex spinor field $z_{\alpha}(\vec{x}, \tau)$, where $\alpha=\uparrow, \downarrow$, and the constraint $\sum_{\alpha}\left|z_{\alpha}\right|^{2}=1$ is obeyed everywhere in spacetime. The Néel order parameter, $\mathbf{n}$ is related to $z_{\alpha}$ by

$$
\mathbf{n}=z_{\alpha}^{\dagger} \vec{\sigma}_{\alpha \beta} z_{\beta},
$$

where $\vec{\sigma}$ are the Pauli matrices. Also, in our analysis, we find it useful to generalize to the $\mathbb{C P}^{N-1}$ model with $\mathrm{SU}(N)$ symmetry, where $\alpha=1 \ldots N$, and then the Pauli matrices are replaced by the generators of $\mathrm{SU}(N)$. The action of the $\mathbb{C P}^{N-1}$ model also involves a non-compact $\mathrm{U}(1)$ gauge field $A_{\mu}$, and is given by

$$
\mathcal{S}_{b}^{z}=\int d \tau \int d^{2} x\left[\frac{1}{g}\left|\left(\partial_{\mu}-i A_{\mu}\right) z_{\alpha}\right|^{2}+\frac{1}{2 e^{2}}\left(\epsilon_{\mu \nu \lambda} \partial_{\nu} A_{\lambda}\right)^{2}\right] .
$$

This theory describes a Néel-ordered phase for $g<g_{c}$, and a spin-gap state with VBS order for $g \geq g_{c}$ (additional Berry phase terms are needed to obtain the four-fold squarelattice symmetry of the VBS order ${ }^{26}$ ). It is crucial to note that, unlike the situation in $1+1$ dimensions ${ }^{27,28}$, the models $\mathcal{S}_{b}^{\text {n }}$ (in Eq. (1.1)) and $\mathcal{S}_{b}^{z}$ are not equivalent to each other in $2+1$ dimensions. This was established in Ref. 29, and is a consequence of the proliferation of 'hedgehog' or 'monopole' defects at the critical point of $\mathcal{S}_{b}^{\text {n}}$; such defects are absent in the $\mathcal{S}_{b}^{z}$ theory.

Now let us add an impurity to the field theory in Eq. (1.7). It was argued in Ref. 30 that the impurity is now represented by a source term for a static charge $Q=2 S$ at $\vec{x}=0$. Thus

$$
\mathcal{S}_{\mathrm{imp}}^{z}=i Q \int d \tau A_{\tau}(\vec{x}=0, \tau)
$$


As before, we are now interested in describing the ground state of $\mathcal{S}_{b}^{z}+\mathcal{S}_{\text {imp }}^{z}$, which we expect carries total spin $S=1 / 2$. However, now the projection onto the state with $S=1 / 2$ cannot be done by the method used for the LGW theory. For $g \geq g_{c}$, we begin with a $S=0$ ground state of $\mathcal{S}_{b}^{z}$, but now don't find that the impurity term in Eq. (1.8) introduces any net spin: the total spin remains at $S=0$ to all orders in perturbation theory. Clearly, we need the impurity charge $Q$ to non-perturbatively bind a $S=1 / 2 z_{\alpha}$ spinon. For $g>g_{c}$, such binding can be addressed via a non-relativistic Schrödinger equation ${ }^{31}$, the analysis does not appear appropriate at the main point of interest, $g=g_{c}$, where we have a conformal field theory (CFT) with no sharp quasiparticle excitations. Here we expect the spinon to be smeared over the whole system of size of $L$. We shall describe this spinon state by explicitly beginning with a $S=1 / 2$ state of $\mathcal{S}_{b}^{z}$ and then perturbatively examining the influence of $\mathcal{S}_{\text {imp }}^{z}$ : this is expected to yield correlations in the true $S=1 / 2$ ground state of $\mathcal{S}_{b}^{z}+\mathcal{S}_{\text {imp }}^{z}$.

Using the language of general $\mathrm{SU}(N)$, let the ground states of $\mathcal{S}_{b}^{z}+\mathcal{S}_{\text {imp }}^{z}$ be $|\alpha\rangle$; these transform under the fundamental representation of $\mathrm{SU}(N)$. To find the matrix element of some operator $O(\vec{x})$ between states $|\alpha\rangle$ and $|\beta\rangle$ of the $\mathrm{SU}(N)$ multiplet, we compute,

$$
\langle\alpha|O(\vec{x})| \beta\rangle=\lim _{\mathcal{T} \rightarrow \infty} \frac{\left\langle z_{\alpha}(0, \mathcal{T} / 2) \exp \left(-i \int_{-\mathcal{T} / 2}^{\mathcal{T} / 2} A_{\tau}(0, \tau) d \tau\right) O(\vec{x}, 0) z_{\beta}^{\dagger}(0,-\mathcal{T} / 2)\right\rangle_{\mathcal{S}_{b}^{z}}}{\left\langle z_{\alpha}(0, \mathcal{T} / 2) \exp \left(-i \int_{-\mathcal{T} / 2}^{\mathcal{T} / 2} A_{\tau}(0, \tau) d \tau\right) z_{\alpha}^{\dagger}(0,-\mathcal{T} / 2)\right\rangle_{\mathcal{S}_{b}^{z}}}
$$

Effectively, we start with external charge free vacuum, and then at time $\tau=-\mathcal{T} / 2$ create a spinon together with the Wilson line, the latter representing the effect of the external charge $Q=1$. We wait for a long time $\mathcal{T} / 2$ to single out the lowest energy state with the quantum numbers of the operator $z_{\alpha}^{\dagger}$. We then measure the operator $O(\vec{x})$, again wait time $\mathcal{T} / 2$ and annihilate our spinon together with the external charge. The denominator in Eq. (1.9) serves to cancel out the matrix element for creating the spinon-external charge bound state out of the vacuum (no sum over $\alpha$ is implied in the denominator). Expressions of type (1.9) are common when studying the properties of heavy-light mesons in quantum chromodynamics.

The time $\mathcal{T}$ must be much larger than the gap between states with the quantum numbers that we are studying. In the spin gap phase, $g>g_{c}$, this gap is finite in the infinite volume limit. However, at the critical point the gap will be of order $1 / L$. So one has to choose $\mathcal{T} \gg L$. Although unusual, this condition can always be satisfied as we work at zero temperature.

To discuss higher charge impurity $(Q>1)$ one needs to act on the vacuum with higher $U(1)$ charge composite operators of the $z$ field. The resulting states can form higher representations of $\mathrm{SU}(N)$ symmetry. For simplicity, we limit ourselves to $Q=1$ below.

Details of our evaluation of Eq. (1.9) in the $1 / N$ expansion appear in Section III A. We will obtain results for the scaling functions appearing in Eq. (1.3) and (1.5) describing the spin distribution at the deconfined quantum critical point.

In addition, in Section III B we compute the uniform and staggered spin distributions in the Néel phase of the $\mathbb{C P}^{N-1}$ model. We find that the short distance behaviour of spin 
distributions both at the critical point and in the Neel phase is in agreement with the impurity scaling theory postulated in Ref. 30. In particular, we obtain substantial additional evidence that the uniform and staggered spin operators flow to the same impurity spin operator upon approaching the impurity site. Results of the $1 / N$ expansion for the impurity critical exponents of uniform and staggered magnetization are obtained.

\section{LGW CRITICALITY}

This section will study the field theory $\mathcal{S}_{b}^{\mathbf{n}}+\mathcal{S}_{\text {imp }}^{\mathbf{n}}$ describing an impurity in an antiferromagnet with an even number of $S=1 / 2$ spins per unit cell. As discussed in Section I, the $\mathrm{O}(3)$ non-linear sigma model formulation in Eqs. (1.1) and (1.2) is not appropriate for our purposes. Instead, we shall use a 'soft-spin' approach which yields a convenient description of the rotationally-invariant state of the bulk antiferromagnet for $g \geq g_{c}$, and of its impurity-induced deformations. The universal results appear in an expansion in

$$
\epsilon=(3-d)
$$

where $d$ is the spatial dimensionality.

This dimensionality expansions allow us to compute, in principle, the universal scaling functions, appearing in Eqs. (1.3) and (1.5), which were numerically computed recently in Ref. 18. The scaling functions clearly depend upon the geometry of the sample, and the nature of the finite-size boundary conditions. Such features are not easily captured in a dimensionality expansion. Consequently the results in this section are more a "proof of principle" that the scaling results apply. Direct comparison of the results below for scaling functions to the numerical results are not very useful.

As discussed in Ref. 10, the $\epsilon$ expansion is obtained by replacing the fixed length field $\mathbf{n}$ by a field $\phi$ whose amplitude is allowed to vary freely. However, we do not have the freedom to relax the length constraint on the impurity site because the Berry phase term is only defined for a unit length field. Consequently, we retain an independent field $\mathbf{n}(\tau)$ representing the impurity spin, which is now linearly coupled to $\phi$. So we consider the theory

$$
\begin{aligned}
\mathcal{Z}_{\phi} & =\int \mathcal{D} \boldsymbol{\phi}(\vec{x}, \tau) \mathcal{D} \mathbf{n}(\tau) \delta\left(\mathbf{n}^{2}-1\right) \exp \left(-\mathcal{S}_{b}^{\phi}-\mathcal{S}_{\text {imp }}^{\phi}\right) \\
\mathcal{S}_{b}^{\phi} & =\int d^{d} x d \tau\left[\frac{1}{2}\left(\left(\partial_{\mu} \boldsymbol{\phi}\right)^{2}+s \phi^{2}\right)+\frac{g_{0}}{4 !}\left(\phi^{2}\right)^{2}\right] \\
\mathcal{S}_{\text {imp }}^{\phi} & =i S \int d \tau \mathbf{A}[\mathbf{n}(\tau)] \cdot \frac{d \mathbf{n}(\tau)}{d \tau}-\gamma_{0} S \mathbf{n}(\tau) \cdot \boldsymbol{\phi}(0, \tau)
\end{aligned}
$$

Here $s \sim g$ is the coupling that tunes the system across the bulk quantum phase transition, and $g_{0}$ and $\gamma_{0}$ are the couplings which were shown in Ref. 10 to approach fixed point values in the vicinity of the quantum critical point. In the $(3-d)$ expansion, these fixed point values are small with $g_{0} \sim \gamma_{0}^{2} \sim \epsilon$. It was argued in Ref. 15 that this fixed point is identical 
to that obtained from the $\mathrm{O}(3)$ non-linear sigma model theory appearing in Eqs. $(1.1,1.2)$.

We will be interested here in the $s \geq s_{c}$ regime of $\mathcal{Z}_{\phi}$ here, where $\langle\phi\rangle=0$ and full rotational symmetry is preserved in the absence of the impurity. As discussed in Section I, we need to project on to the state with total $S_{z}=1 / 2$ in the presence of the impurity. This is easily done here by choosing the following parameterization for the impurity degree of freedom $\mathbf{n}(\tau)$ in terms of a complex scalar $\psi(\tau)$ :

$$
\mathbf{n}=\left(\frac{\psi+\psi^{*}}{2} \sqrt{2-|\psi|^{2}}, \frac{\psi-\psi^{*}}{2 i} \sqrt{2-|\psi|^{2}}, 1-|\psi|^{2}\right) \text {. }
$$

The advantage of the representation (2.3) is that with the gauge choice

$$
\mathbf{A}(\mathbf{n})=\frac{1}{1+n_{z}}\left(-n_{y}, n_{x}, 0\right)
$$

the Berry phase takes the following form

$$
i \mathbf{A}(\mathbf{n}) \cdot \frac{d \mathbf{n}}{d \tau}=\frac{1}{2}\left(\psi^{*} \frac{\partial \psi}{\partial \tau}-\psi \frac{\partial \psi^{*}}{\partial \tau}\right)
$$

Furthermore, the measure term in the functional integral also has the simple form

$$
\int \mathcal{D} \mathbf{n} \delta\left(\mathbf{n}^{2}-1\right)=\int \mathcal{D} \psi \mathcal{D} \psi^{*}
$$

Now, an expansion of the correlators of $\mathcal{Z}_{\phi}$, in a functional integral over $\phi$ and $\psi$ about the saddle point with $\phi=0$ and $\psi=0$, in powers of the couplings $\gamma_{0}$ and $g_{0}$, automatically projects onto the state with total spin projection $S_{z}=1 / 2$. This is easily established by applying a uniform magnetic field, and verifying by the methods of Ref. 10,15 that the total magnetization is quantized by a Ward identity associated with the conservation of spin.

We can now use the above perturbative expansion, using methods explained at length elsewhere ${ }^{10,15}$, to compute the expectation values of the magnetization density $\left\langle Q_{z}(\vec{x})\right\rangle$ and the Néel order parameter $\left\langle\phi_{z}(\vec{x})\right\rangle$. We perform this computation on a sample with periodic boundary conditions and length $L$ in each spatial dimension, i.e. a torus $T^{d}$. The main effect of the finite boundary conditions is that the momenta $\vec{p}$ are discrete, and each momentum component is quantized in integer multiples of $2 \pi / L$. The results below are easily generalized to other finite size geometries and boundary conditions. To leading order in $\epsilon$, the results are

$$
\begin{aligned}
\left\langle Q_{z}(\vec{x})\right\rangle & =S \delta^{d}(\vec{x})-\gamma_{0}^{2} S \delta^{d}(\vec{x}) \int \frac{d \omega}{2 \pi} \frac{1}{(i \omega+\varepsilon)^{2}} G(\omega, 0)+2 \gamma_{0}^{2} S \int \frac{d \omega}{2 \pi} G(\omega, \vec{x}) G(\omega,-\vec{x}) \\
\left\langle\phi_{z}(\vec{x})\right\rangle & =\gamma_{0} S G(0, \vec{x})\left[1-\gamma_{0}^{2} \int \frac{d \omega}{2 \pi} \frac{1}{(i \omega+\varepsilon)^{2}} G(\omega, 0)\right]
\end{aligned}
$$

where $\varepsilon$ is a positive infinitesimal proportional to an applied magnetic field which selects 
the $S_{z}=1 / 2$ state. We may set $\varepsilon=0$ after the frequency integrals have been performed. The Green's function of the $\phi$ field is

$$
G(\omega, \vec{x})=\frac{1}{L^{d}} \sum_{\vec{p}} \frac{e^{i \vec{p} \cdot \vec{x}}}{\omega^{2}+\vec{p}^{2}+\Delta^{2}},
$$

where $\Delta$ is the spin gap of the bulk antiferromagnet in the absence of the impurity. Other boundary conditions will only change the form of $G$. It is easy to check that the spatial integral of $\left\langle Q_{z}\right\rangle$ is quantized at $S$.

To leading order in $\epsilon$, it would appear that we can set $\Delta$ equal to the spin gap in the infinite bulk antiferromagnet, and in particular, set $\Delta=0$ at the critical point $s=s_{c}$. However, we will see below that for the particular boundary conditions we are using here, there are infrared divergencies at $\Delta=0$ in the expressions for the impurity-induced spin textures. In such a situation we have to examine the finite $L$ corrections to the value of $\Delta$ at $s=s_{c}$, which yield a non-zero $\Delta$ even at the bulk quantum critical point. The value of $\Delta$ can be computed as described elsewhere ${ }^{32}$, and to leading order in $\epsilon$, the equation determining $\Delta$ at the quantum critical point $s=s_{c}$ is

$$
\Delta^{2}=\frac{5 g_{0}}{6} \frac{1}{L^{d}} \sum_{\vec{p}} \int \frac{d \omega}{2 \pi} \frac{1}{\omega^{2}+\vec{p}^{2}+\Delta^{2}}
$$

To leading order in $\epsilon$, only the $\vec{p}=0$ term on the right-hand-side has to be included; setting $g_{0}$ equal to its fixed point value ${ }^{32}$ we find for small $\epsilon$

$$
\Delta=\left(\frac{20 \pi^{2} \epsilon}{11}\right)^{1 / 3} \frac{1}{L}
$$

Note that $L \Delta$ is a universal number at $s=s_{c}$, which is the main result we will need below to establish the universality of the spin texture.

Returning to the expressions in Eq. (2.7), we now want to manipulate them into the forms of Eq. (1.3) and (1.5). However, the presence of the $\delta^{d}(\vec{x})$ in Eq. (2.7) makes the $\vec{x}$ dependence singular. These singularities are in fact an artifact of the present perturbative expansion in real space, and are not expected to be present once the expansion is resummed. This is evident by examining the results in momentum space, where the results are a smooth function of momentum. In this manner we obtain after applying Eq. (2.8) to Eq. (2.7)

$$
\begin{aligned}
& \left\langle Q_{z}(\vec{p})\right\rangle=S\left[1-\frac{\gamma_{0}^{2}}{L^{d}} \sum_{\vec{q}} \frac{1}{2 E_{\vec{q}}}\left(\frac{1}{E_{\vec{q}}^{2}}-\frac{2}{E_{\vec{p}+\vec{q}}\left(E_{\vec{p}+\vec{q}}+E_{\vec{q}}\right)}\right)\right] \\
& \left\langle\phi_{z}(\vec{p})\right\rangle=\frac{\gamma_{0} S}{\vec{p}^{2}+\Delta^{2}}\left[1-\frac{\gamma_{0}^{2}}{L^{d}} \sum_{\vec{q}} \frac{1}{2 E_{\vec{q}}^{3}}\right]
\end{aligned}
$$


where $E_{\vec{p}}=\sqrt{\vec{p}^{2}+\Delta^{2}}$. Now Eqs. (2.11) can be evaluated at the fixed point value of $\gamma_{0}$, and to leading order in $\epsilon$ they are seen to yield results consistent with the following scaling forms which can be deduced from Eqs. (1.3,1.5)

$$
\begin{aligned}
\left\langle Q_{z}(\vec{p})\right\rangle & =\widetilde{\Phi}_{Q}(\vec{p} L) \\
\left\langle\phi_{z}(\vec{p})\right\rangle & =L^{(d+1-\eta) / 2} \widetilde{\Phi}_{n}(\vec{p} L)
\end{aligned}
$$

The explicit results for the scaling functions to leading order in $\epsilon$ are

$$
\begin{aligned}
& \widetilde{\Phi}_{Q}(\vec{y})=S\left[1-2 \pi^{2} \epsilon \sum_{\vec{x}} \frac{1}{2 \mathcal{E}_{\vec{x}}}\left(\frac{1}{\mathcal{E}_{\vec{x}}^{2}}-\frac{2}{\mathcal{E}_{\vec{y}+\vec{x}}\left(\mathcal{E}_{\vec{y}+\vec{x}}+\mathcal{E}_{\vec{x}}\right)}\right)\right] \\
& \widetilde{\Phi}_{n}(\vec{y})=\frac{\pi S \sqrt{2 \epsilon}}{\vec{y}^{2}+L^{2} \Delta^{2}}\left[1-2 \pi^{2} \epsilon(\text { a finite number })\right]
\end{aligned}
$$

where now $\vec{x}$ and $\vec{y}$ are three dimensional momenta whose components are quantized in integer multiples of $2 \pi$ (except in the integral in the second equation), and $\mathcal{E}_{\vec{x}}=\sqrt{\vec{x}^{2}+L^{2} \Delta^{2}}$. It is easily checked that these expressions are free of infrared and ultraviolet divergencies, and so yield universal results because $L \Delta$ is a universal number.

From the above expression, we observe that $\widetilde{\Phi}_{Q}(|\vec{y}| \rightarrow \infty)=S(1-(\epsilon / 2) \ln |\vec{y}|)$, which we assume exponentiates to $\widetilde{\Phi}_{Q}(|\vec{y}| \rightarrow \infty) \sim|\vec{y}|^{-\epsilon / 2}$. From the short distance behavior of the spin texture discussed in Ref. 18 , we expect that $\widetilde{\Phi}_{Q}(|\vec{y}| \rightarrow \infty) \sim|\vec{y}|^{-\eta^{\prime} / 2}$, where $\eta^{\prime}$ is the scaling dimension of the boundary $\operatorname{spin}^{10}$. So we obtain the value $\eta^{\prime}=\epsilon$, which is consistent with earlier results ${ }^{10}$. Similarly, from the short distance behavior discussed in Ref. 18, we also have $\widetilde{\Phi}_{n}(|\vec{y}| \rightarrow \infty) \sim|\vec{y}|^{-2+\left(\epsilon+\eta-\eta^{\prime}\right) / 2}$. So with $\eta \sim \mathcal{O}\left(\epsilon^{2}\right)$ and $\eta^{\prime}=\epsilon$, we have $\widetilde{\Phi}_{n}(|\vec{y}| \rightarrow \infty) \sim|\vec{y}|^{-2}$, which is consistent with Eq. (2.13).

\section{DECONFINED CRITICALITY}

This section describes the Néel-VBS transition in square lattice quantum antiferromagnets with a single $S=1 / 2$ per unit cell. As discussed in Section I, the response of a non-magnetic impurity is described by the action $\mathcal{S}_{b}^{z}+\mathcal{S}_{\text {imp }}^{z}$ in Eqs. $(1.7,1.8)$ for a complex $\mathrm{SU}(N)$ spinon field $z_{\alpha}$ and a non-compact $\mathrm{U}(1)$ gauge field $A_{\mu}$. Here we will describe the $1 / N$ expansion of its universal critical properties. Note that in what follows we have rescaled the spinon field $z$, to remove the coupling constant $g$ from the action (1.7), in favour of a rescaled constraint $z_{\alpha}^{\dagger} z_{\alpha}=1 / \mathrm{g}$. This constraint is enforced with a local Lagrange multiplier $\lambda$, so that the bulk action becomes,

$$
\mathcal{S}_{b}^{z}=\int d \tau \int d^{2} x\left[\left|\left(\partial_{\mu}-i A_{\mu}\right) z_{\alpha}\right|^{2}+i \lambda\left(\left|z_{\alpha}\right|^{2}-\frac{1}{g}\right)+\frac{1}{2 e^{2}}\left(\epsilon_{\mu \nu \lambda} \partial_{\nu} A_{\lambda}\right)^{2}\right] .
$$

It is useful to define $\mathrm{SU}(N)$ generalizations of the $\mathrm{SU}(2)$ observables introduced in Section I. The uniform magnetization density $\mathbf{Q}$ generalizes to $Q^{a}$, which is the temporal 
component of a current associated with the $\mathrm{SU}(N)$ rotation symmetry,

$$
Q^{a}=z^{\dagger} T^{a} D_{\tau} z-\left(D_{\tau} z\right)^{\dagger} T^{a} z
$$

(where $D_{\mu}=\partial_{\mu}-i A_{\mu}$ is the covariant derivative) while the Néel order $\mathbf{n}$ in Eq. (1.6) becomes the staggered magnetization operator

$$
n^{a}=z^{\dagger} T^{a} z
$$

where $T^{a}$ are generators of the $\mathrm{SU}(N)$ algebra. We will describe the spatial dependence of the expectation values of these operators for two cases: a finite system of size $L$ at the critical point $g=g_{c}$ in Section III A, and the infinite system in the Néel phase with broken $\mathrm{SU}(N)$ symmetry in Section III B.

\section{A. Critical point in a finite system}

We tune the system to the critical point $g=g_{c}$ of the infinite volume zero temperature model, and then consider the system on a spatial torus of length $L$. We use periodic boundary conditions for all fields. ${ }^{2}$ As we discussed in Section I, the ground state in the absence of an impurity is a spin-singlet, while adding an impurity yields a ground state which transforms under the fundamental representation of $\mathrm{SU}(N)$. This ground state has a single spinon in it, and we argued that the projection onto this state can be performed by Eq. (1.9). For an additional test of our projection formalism, see the appendix, where we compute the $U(1)$ (electric) charge density in the presence of the impurity.

Before we address the explicit computation of (1.9), we discuss scaling forms that our results should obey.

\section{Scaling Forms}

We are interested in computing the uniform and staggered magnetization densities. Recall, that since the uniform magnetization is a zeroth component of a conserved current, it receives no renormalizations. Therefore, utilizing the $\mathrm{SU}(N)$ symmetry, we have the general scaling form,

$$
\left\langle\alpha\left|Q^{a}(\vec{x})\right| \beta\right\rangle=\frac{1}{L^{2}} \Phi_{Q}\left(\frac{\vec{x}}{L}\right) T_{\alpha \beta}^{a}
$$

\footnotetext{
${ }^{2}$ In principle, on a spatial torus, we can certainly have a finite magnetic $\left(F_{i j}\right)$ flux, which would correspond to non-periodic boundary conditions. However, finite flux sectors are expected to be separated from vacuum by an energy gap, and hence are suppressed at $T=0$.
} 
The leading $1 / L^{2}$ prefactor corresponds to the scaling dimension $\Delta_{Q}=d=2$ of the magnetization density. Moreover, by conservation of total $\mathrm{SU}(N)$ charge,

$$
\int d^{2} r \Phi_{Q}(\vec{r})=-1
$$

where the integral is over $0<r_{1}, r_{2}<1$. Similarly, for the case of the staggered magnetization,

$$
\left\langle\alpha\left|n^{a}(\vec{x})\right| \beta\right\rangle=\Lambda^{\eta_{n}}\left(\frac{1}{L}\right)^{1-\eta_{n}} \Phi_{n}\left(\frac{\vec{x}}{L}\right) T_{\alpha \beta}^{a}
$$

Here $\eta_{n}$ is the anomalous dimension of the staggered magnetization operator $n^{a}(x), \Delta_{n}=$ $\operatorname{dim}\left[n^{a}\right]=1-\eta_{n}$. This exponent is related to the exponent $\eta$ in Eq. (1.5), and their values were computed previously ${ }^{33}$ in the $1 / N$ expansion for arbitrary spacetime dimension $2<D<4$ :

$$
\eta_{n}=\frac{1}{2}(D-2-\eta)=\frac{1}{N} \frac{16 \Gamma(D-2)}{\Gamma(2-D / 2) \Gamma(D / 2-1)^{3}}+O\left(1 / N^{2}\right) \stackrel{D=3}{=} \frac{16}{\pi^{2} N}+O\left(1 / N^{2}\right) .
$$

The function $\Phi_{Q}$ is completely universal, whereas $\Phi_{n}$ is universal only up to an overall scale. In particular, $\Phi_{n}$ does not have any property analogous to (3.5).

Of particular interest is the behavior of the functions $\Phi_{Q}(\vec{r}), \Phi_{n}(\vec{r})$ for $\vec{r} \rightarrow 0$. We make a hypothesis that $n^{a}(\vec{x}, \tau)$ and $Q^{a}(\vec{x}, \tau)$ flow to the same operator $S^{a}(\tau)$ as $\vec{x}$ approaches the Wilson line,

$$
\begin{aligned}
\lim _{|\vec{x}| \rightarrow 0} Q^{a}(\vec{x}, \tau) & =\frac{c_{Q}}{|\vec{x}|^{-\Delta_{\text {imp }}^{Q}}} S^{a}(\tau) \\
\lim _{|\vec{x}| \rightarrow 0} n^{a}(\vec{x}, \tau) & =\frac{c_{n}}{|\vec{x}|^{-\Delta_{\text {imp }}^{n}}} S^{a}(\tau)
\end{aligned}
$$

Calculations in the $\epsilon$ expansion supporting this hypothesis have been given in Ref. 30. We have performed analogous calculations in the $1 / N$ expansion again confirming the OPE (3.8). Technically, this impurity OPE program consists of the following steps. First one considers the (multiplicative) renormalization of the operator $n^{a}(\vec{x}=0)$, by studying its insertion into the two point function of the $z$ field (this consist of the usual bulk renormalization, plus an additional renormalization of the logarithmic divergences that appear as $\vec{x} \rightarrow 0$ ). Once $n^{a}(\vec{x}=0)$ operator is renormalized, one considers the insertion of $Q^{a}(\vec{x} \rightarrow 0)$ into the two point function of the $z$ field. The highest divergence as $|\vec{x}| \rightarrow 0$ is power-like, $1 /|\vec{x}|$, modified by logarithms at higher orders in $1 / N$. This leading divergence can be cancelled by a $n^{a}(\vec{x}=0$ ) counterterm (with a coefficient that diverges as $\vec{x} \rightarrow 0$ ). This procedure gives one a way to construct order by order in $1 / N$, the impurity operator $S^{a}(\tau)$ (which is essentially a regularized $n^{a}(\vec{x}=0, \tau)$ ), and compute the anomalous dimensions $\Delta_{\text {imp }}^{Q}$, $\Delta_{\text {imp }}^{n}$ as well as coefficients $c_{Q}, c_{n}$ (the later are renormalization scheme dependent). As the computation of the OPE in the $1 / N$ expansion essentially follows that in the $\epsilon$ expansion presented in Ref. 30, we shall not include it here. We only note that in this way, we have 
been able to explicitly check the OPE (3.8) to order $1 / N^{2}$, obtaining $\Delta_{\text {imp }}^{n}$ to order $1 / N^{2}$ and $\Delta_{\text {imp }}^{Q}$ to order $1 / N$ (this is lower order than the corresponding result for $\Delta_{\text {imp }}^{n}$ as $c_{Q} / c_{n}$ is of order $1 / N)$. Explicit results in this expansion will appear in Section III B.

Calculations of $\Phi_{Q}$ and $\Phi_{n}$ given below provide additional support for the OPE (3.8). Note that the exponents $\Delta_{\text {imp }}^{Q}$ and $\Delta_{\text {imp }}^{n}$ are not independent. Indeed, let the correlator

$$
\left\langle S^{a}(\tau) S^{b}(0)\right\rangle \sim \frac{1}{\tau^{2 \Delta_{S}}} \delta_{a b} .
$$

The exponent $\Delta_{S}$ is related to the boundary spin exponent $\eta^{\prime}$ used in Refs. 10,18 by $\eta^{\prime}=2 \Delta_{S}$. Then,

$$
\Delta_{S}=\Delta_{Q}+\Delta_{\mathrm{imp}}^{Q}=\Delta_{n}+\Delta_{\mathrm{imp}}^{n}
$$

Recalling, $\Delta_{Q}=2, \Delta_{n}=1-\eta_{n}$,

$$
\Delta_{\mathrm{imp}}^{Q}=\Delta_{\mathrm{imp}}^{n}-1-\eta_{n} .
$$

Our explicit results for the profiles $\Phi_{Q}, \Phi_{n}$ confirm the relation (3.11) to leading (zeroth) order in $1 / N$, see below. We have also been able to check this relation to order $1 / N$ using the impurity OPE program summarized above: to this order, $\Delta_{\text {imp }}^{Q}=-1-\eta_{n}$, as $\Delta_{\text {imp }}^{n} \sim O\left(1 / N^{2}\right)$. The result of our evaluation of $\Delta_{\text {imp }}^{n}$ to $O\left(1 / N^{2}\right)$ will appear later in Eqs. (3.98),(3.99).

Note that the OPE (3.8) is sensitive only to short distance physics, and, thus, coefficients $c_{Q}, c_{n}$ should be independent of the system size $L$ as well as the deviation from the critical point (all this IR information is, however, contained in the impurity operator $S^{a}$ ). Thus, the ratio,

$$
\frac{c_{Q}}{c_{n}}=\lim _{|\vec{x}| \rightarrow 0}|\vec{x}|^{\Delta_{\operatorname{imp}}^{n}-\Delta_{\operatorname{imp}}^{Q}} \frac{\left\langle Q^{a}(\vec{x})\right\rangle}{\left\langle n^{a}(\vec{x})\right\rangle}=\lim _{|\vec{x}| \rightarrow 0}|\vec{x}|^{1+\eta_{n}} \frac{\left\langle Q^{a}(\vec{x})\right\rangle}{\left\langle n^{a}(\vec{x})\right\rangle}
$$

although non-universal, should be constant throughout the scaling regime (once the regularization scheme is chosen). We shall check this fact below to leading order in $1 / N$ by comparing the short distance behaviour (controlled by the OPE) of uniform and staggered magnetization densities at the critical point and in the Néel phase.

\section{Projection onto the Single Spinon State}

Now we return to the evaluation of the matrix elements (1.9). Although it is possible to obtain all the results presented below directly from Eq. (1.9) it is technically somewhat simpler to use instead,

$$
\langle\alpha|O(\vec{x})| \beta\rangle=\lim _{\mathcal{T} \rightarrow \infty} \frac{\left\langle z_{\alpha}(\vec{k}, \mathcal{T} / 2) O(\vec{x}, 0) z_{\beta}^{\dagger}\left(\vec{k}^{\prime},-\mathcal{T} / 2\right)\right\rangle_{\text {imp }}}{\left\langle z_{\alpha}(\vec{k}, \mathcal{T} / 2) z_{\alpha}^{\dagger}\left(\vec{k}^{\prime},-\mathcal{T} / 2\right)\right\rangle_{\text {imp }}}
$$


Here, $z_{\alpha}(\vec{k}, \tau)=\int d^{2} x z_{\alpha}(\vec{x}, \tau) e^{-i \vec{k} \vec{x}}$ and the subscript "imp" indicates that the correlator should be computed in a theory with the action $\mathcal{S}_{b}^{z}+\mathcal{S}_{\text {imp }}^{z}$ which includes the impurity term. Effectively, we have extended the Wilson line, which in (1.9) stretched from the point where a spinon was created to the point where it was destroyed, to run from $\tau=-\infty$ to $\tau=\infty$. In addition, we have taken our "incoming" and "outgoing" spinon to be in momenta $\vec{k}$ and $\vec{k}^{\prime}$ states. This makes the numerator and denominator of (3.13) non-gauge invariant. Nevertheless, we expect that this non-gauge invariance comes solely from the matrix element for creating the ground state of the system by acting on the vacuum with $z^{\dagger}$ and cancels out between the numerator and denominator of (3.13).

Since the impurity term Eq. (1.8) breaks spatial (but not temporal) translational invariance, for $\mathcal{T} \rightarrow \infty$ we expect to obtain the ground state irrespective of which $\vec{k}, \vec{k}^{\prime}$ we started with. Nevertheless, it will be most convenient in our perturbative treatment to work with $\vec{k}=\vec{k}^{\prime}=0$.

Since the external charge does not break $\mathrm{SU}(N)$ symmetry and time translation symmetry, we have,

$$
\left\langle z_{\alpha}(x) z_{\beta}^{\dagger}\left(x^{\prime}\right)\right\rangle_{\mathrm{imp}}=\delta_{\alpha \beta} D\left(\vec{x}, \vec{x}^{\prime}, \tau-\tau^{\prime}\right)
$$

We let,

$$
D\left(\vec{x}, \vec{x}^{\prime}, \tau\right)=\frac{1}{L^{2}} \sum_{\vec{p}, \vec{p}^{\prime}} \int \frac{d \omega}{2 \pi} D\left(\vec{p}, \vec{p}^{\prime}, \omega\right) e^{i \vec{p} \vec{x}} e^{-i \vec{p}^{\prime} \vec{x}^{\prime}} e^{i \omega \tau}
$$

We write,

$$
\left\langle z_{\alpha}(y) O(x) z_{\beta}^{\dagger}\left(y^{\prime}\right)\right\rangle_{\mathrm{imp}}=\int d v d v^{\prime} D(y, v) O_{\alpha \beta}\left(v, x, v^{\prime}\right) D\left(v^{\prime}, y^{\prime}\right)
$$

Fourier transforming,

$O_{\alpha \beta}\left(v, x, v^{\prime}\right)=\frac{1}{L^{2}} \sum_{\vec{p}} \frac{1}{L^{2}} \sum_{\vec{p}^{\prime}} \int \frac{d \omega}{2 \pi} \int \frac{d \omega^{\prime}}{2 \pi} O_{\alpha \beta}\left(\vec{p}, \vec{q}, \vec{p}^{\prime}, \omega, \omega^{\prime}\right) e^{i \vec{p} \vec{v}} e^{-i \vec{p}^{\prime} \vec{v}^{\prime}} e^{i \vec{q} \vec{x}} e^{i \omega v_{\tau}} e^{-i \omega^{\prime} v_{\tau^{\prime}}} e^{i\left(\omega^{\prime}-\omega\right) x_{\tau}}$

where we use the notation that the three-vector $x$ has spatial components $\vec{x}$ and temporal component $x_{\tau}$. So,

$$
\begin{aligned}
& \left\langle z_{\alpha}(\vec{k}, \mathcal{T} / 2) O(\vec{x}, 0) z_{\beta}^{\dagger}\left(\vec{k}^{\prime},-\mathcal{T} / 2\right)\right\rangle_{\mathrm{imp}}= \\
& \sum_{\vec{p}, \vec{p}^{\prime}, \vec{q}} \int \frac{d \omega}{2 \pi} \frac{d \omega^{\prime}}{2 \pi} D(\vec{k}, \vec{p}, \omega) O_{\alpha \beta}\left(\vec{p}, \vec{q}, \vec{p}^{\prime}, \omega, \omega^{\prime}\right) D\left(\vec{p}^{\prime}, \vec{k}^{\prime}, \omega^{\prime}\right) e^{i \omega \mathcal{T} / 2} e^{i \omega^{\prime} \mathcal{T} / 2} e^{i \vec{q} \vec{x}}
\end{aligned}
$$

As we perform the integral over $\omega, \omega^{\prime}$, we pick up poles of the propagators $D$ in the $\Im(\omega)>0$, $\Im\left(\omega^{\prime}\right)>0$ planes (we expect that $O_{\alpha \beta}$ is analytic in $\omega$ ). In the limit $\mathcal{T} \rightarrow \infty$ only the contribution from the pole with smallest imaginary part survives. Let this pole be at $\omega=i m$ 
and denote by $\operatorname{Res}(\vec{k}, \vec{p})$ the residue of $D(\vec{k}, \vec{p}, \omega)$ at this pole. Then,

$$
\left\langle z_{\alpha}(\vec{k}, \mathcal{T} / 2) O(\vec{x}, 0) z_{\beta}^{\dagger}\left(\vec{k}^{\prime},-\mathcal{T} / 2\right)\right\rangle_{\mathrm{imp}} \rightarrow \sum_{\vec{p}, \vec{p}^{\prime}, \vec{q}}(i \operatorname{Res}(\vec{k}, \vec{p}))\left(i \operatorname{Res}\left(\vec{p}^{\prime}, \vec{k}^{\prime}\right)\right) O_{\alpha \beta}\left(\vec{p}, \vec{q}, \vec{p}^{\prime}, i m, i m\right) e^{i \vec{q} \vec{x}} e^{-m \mathcal{T}}
$$

Similarly, the denominator of (3.13) is,

$$
\left\langle z_{\alpha}(\vec{k}, \mathcal{T} / 2) z_{\alpha}^{\dagger}\left(\vec{k}^{\prime},-\mathcal{T} / 2\right)\right\rangle_{\mathrm{imp}} \rightarrow L^{2} i \operatorname{Res}\left(k, k^{\prime}\right) e^{-m \mathcal{T}}
$$

Finally,

$$
\langle\alpha|O(\vec{x})| \beta\rangle=\frac{1}{L^{2}} \sum_{q}\langle\alpha|O(\vec{q})| \beta\rangle e^{i \vec{q} \vec{x}}
$$

with,

$$
\langle\alpha|O(\vec{q})| \beta\rangle=\sum_{\vec{p}, \vec{p}^{\prime}} \frac{(i \operatorname{Res}(\vec{k}, \vec{p}))\left(i \operatorname{Res}\left(\vec{p}^{\prime}, \vec{k}^{\prime}\right)\right)}{i \operatorname{Res}\left(k, k^{\prime}\right)} O_{\alpha \beta}\left(\vec{p}, \vec{q}, \vec{p}^{\prime}, i m, i m\right)
$$

\section{Large $N$ expansion of $\mathbb{C P}^{N-1}$ theory in finite volume}

We now compute the expression (3.22) using the large $N$ expansion in finite volume. First, consider the $N=\infty$ limit. The gap equation reads,

$$
\frac{1}{L^{2}} \sum_{\vec{p}} \int \frac{d \omega}{2 \pi} \frac{1}{\omega^{2}+\vec{p}^{2}+m_{0}^{2}}=\frac{1}{g N}
$$

and to this order in $N, m_{0}^{2}=i\langle\lambda\rangle$. In the infinite volume, the critical coupling $g=g_{c}$ is obtained when the gap $m_{0}$ vanishes,

$$
\frac{1}{g_{c} N}=\int \frac{d^{3} p}{(2 \pi)^{3}} \frac{1}{p^{2}}
$$

However, once we make the spatial volume finite, a non-zero $m_{0}$ is generated even at the critical point. Thus, setting $g=g_{c}$, using Eq. (3.24) and poisson resumming, we obtain,

$$
\sum_{\vec{n} \in \mathbf{Z}^{2}} \int \frac{d \omega}{2 \pi} \int \frac{d^{2} p}{(2 \pi)^{2}} e^{i \vec{p} \vec{n} L} \frac{1}{\omega^{2}+\vec{p}^{2}+m_{0}^{2}}=\int \frac{d \omega}{2 \pi} \int \frac{d^{2} p}{(2 \pi)^{2}} \frac{1}{\omega^{2}+\vec{p}^{2}}
$$

On the left-hand side, only the $\vec{n}=0$ term diverges in the UV. However, this divergence cancels with the divergence of the right-hand side. Thus, performing all integrals,

$$
\sum_{\vec{n} \neq 0} \frac{1}{4 \pi|\vec{n}|} e^{-m_{0}|\vec{n}| L}=\frac{m_{0} L}{4 \pi}
$$


The solution of the Eq. (3.26) is,

$$
m_{0}=\theta \frac{1}{L}
$$

where $\theta$ is a constant that can be obtained by solving (3.26) numerically to be, $\theta \approx 1.51196$.

Thus, at leading order the propagator,

$$
D_{0}\left(\vec{k}, \vec{k}^{\prime}, \omega\right)=\delta_{\vec{k}, \vec{k}^{\prime}} \frac{1}{\omega^{2}+\vec{k}^{2}+m_{0}^{2}}
$$

and the lowest pole is at $\vec{k}=0, \omega=i m_{0}$ and, $i \operatorname{Res}(\vec{k}, \vec{p})=\delta_{\vec{k}, 0} \delta_{\vec{p}, 0} \frac{1}{2 m_{0}}$.

To develop the $1 / N$ expansion, we will need to find the $A_{\mu}$ and $\lambda$ propagators. The dynamically generated self-energy for $A_{\mu}$ is to leading order,

$$
K_{\mu \nu}(p)=-N \frac{1}{L^{2}} \sum_{\vec{q}} \int \frac{d q_{\tau}}{2 \pi}\left(\frac{(2 q-p)_{\mu}(2 q-p)_{\nu}}{\left((q-p)^{2}+m_{0}^{2}\right)\left(q^{2}+m_{0}^{2}\right)}-\frac{2 \delta_{\mu \nu}}{\left(q^{2}+m_{0}^{2}\right)}\right)
$$

This self energy is always more singular near the critical point than the bare Maxwell term in $\mathcal{S}_{b}^{z}$, and so we will work with $e^{2}=\infty$ for the rest of this paper. To find the photon propagator, $\mathcal{D}_{\mu \nu}(p)$, we also need to fix a gauge. Practically, for the calculations to follow, we will only need the static electromagnetic propagator $\mathcal{D}_{\tau \tau}\left(\vec{p}, p_{\tau}=0\right)=K_{\tau \tau}\left(\vec{p}, p_{\tau}=0\right)^{-1}$, which is a gauge invariant quantity. We also note that in the infinite volume limit,

$$
\begin{aligned}
K_{\mu \nu}(q) & =K(q)\left(q^{2} \delta_{\mu \nu}-q_{\mu} q_{\nu}\right) \\
K(q) & =N A q^{D-4}
\end{aligned}
$$

where the constant $A$ is given by,

$$
A=\frac{1}{(4 \pi)^{D / 2}} \frac{(D-2) \Gamma(2-D / 2) \Gamma(D / 2-1)^{2}}{\Gamma(D)}
$$

Here $D$ is the space-time dimension. In our case, $D=3$ and $A=\frac{1}{16}$.

Likewise, the self-energy for $\lambda$ is to leading order,

$$
\Pi(p)=N \frac{1}{L^{2}} \sum_{\vec{q}} \int \frac{d q_{\tau}}{2 \pi} \frac{1}{\left(q^{2}+m_{0}^{2}\right)\left((q-p)^{2}+m_{0}^{2}\right)}
$$

In the infinite volume limit,

$$
\Pi(p)=N B p^{D-4}
$$

where the constant $B$ is given by,

$$
B=\frac{1}{(4 \pi)^{D / 2}} \frac{\Gamma(2-D / 2) \Gamma(D / 2-1)^{2}}{\Gamma(D-2)}
$$


FIG. 1: The insertion of $Q^{a}$ into the $z$ propagator.

For $D=3, B=\frac{1}{8}$.

\section{Matrix Elements}

Now, let us compute the matrix elements of operator $Q^{a}(x)$. The insertion of $Q^{a}$ into the $z$ propagator, to leading order in $1 / N$ is given by diagram in Fig. 1 , so

$$
Q_{\alpha \beta}^{a}\left(\vec{p}, \vec{q}, \vec{p}^{\prime}, \omega, \omega^{\prime}\right)=i\left(\omega+\omega^{\prime}\right) T_{\alpha \beta}^{a} \delta_{\vec{q}, \vec{p}^{\prime}-\vec{p}}
$$

So utilizing formula (3.22), with $\vec{k}=\vec{k}^{\prime}=0$, we obtain,

$$
\left\langle\alpha\left|Q^{a}(\vec{q})\right| \beta\right\rangle=-T_{\alpha \beta}^{a} \delta_{\vec{q} 0}
$$

i.e.,

$$
\left\langle\alpha\left|Q^{a}(\vec{x})\right| \beta\right\rangle=-\frac{1}{L^{2}} T_{\alpha \beta}^{a}
$$

and the function $\Phi_{Q}(\vec{r})=-1$, satisfies the normalization condition (3.5). So at leading order in the $1 / N$ expansion the magnetization in the presence of an impurity is spatially uniform. The system with the impurity simply consists of a free spinon in the zero momentum state. The effects of the interaction with the impurity appear only at next order in $1 / N$.

Similarly, for the staggered magnetization, the insertion of $n^{a}(x)$ into the $z$ propagator, to leading order is given by the same diagram in Fig. 1, except the cross now stands for $n^{a}$.

$$
n_{\alpha \beta}^{a}\left(\vec{p}, \vec{q}, \vec{p}^{\prime}, \omega, \omega^{\prime}\right)=\delta_{\vec{q}, \vec{p}^{\prime}-\vec{p}} T_{\alpha \beta}^{a}
$$

so that,

$$
\left\langle\alpha\left|n^{a}(\vec{q})\right| \beta\right\rangle=\frac{1}{2 m_{0}} \delta_{\vec{q} 0} T_{\alpha \beta}^{a}
$$

and,

$$
\left\langle\alpha\left|n^{a}(\vec{x})\right| \beta\right\rangle=\frac{1}{2 \theta L} T_{\alpha \beta}^{a}
$$

So the staggered magnetization at leading order in $1 / N$ is also uniform, $\Phi_{n}(\vec{r})=\frac{1}{2 \theta}$.

Now, let's include the $1 / N$ corrections.

We will concentrate on corrections to $\langle\alpha|O(\vec{q})| \beta\rangle$, for $O=Q^{a}$, $n^{a}$, with $\vec{q} \neq 0$ (where the leading $\mathrm{O}(1)$ term vanishes). These turn out to be much simpler to compute than corrections for $\vec{q}=0$. Moreover, for $Q^{a}$, we know by $\operatorname{SU}(N)$ charge conservation that the $N=\infty$ result 

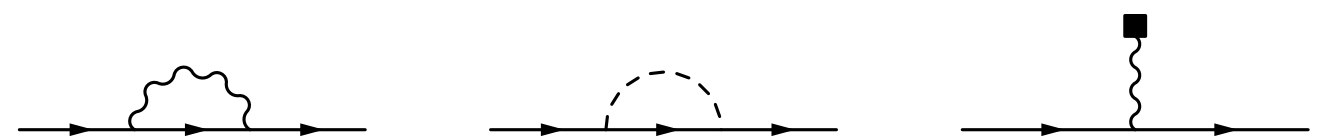

FIG. 2: $1 / N$ corrections to $z$ self-energy.

(3.37) at $\vec{q}=0$ receives no further corrections. Thus, to order $1 / N$,

$$
\begin{aligned}
\langle\alpha|O(\vec{q})| \beta\rangle \stackrel{\vec{q} \neq 0}{=} i \operatorname{Res}(0,-\vec{q})_{1} O_{\alpha \beta}\left(-\vec{q}, \vec{q}, 0, i m_{0}, i m_{0}\right)_{0}+i \operatorname{Res}(\vec{q}, 0)_{1} O_{\alpha \beta}\left(0, \vec{q}, \vec{q}, i m_{0}, i m_{0}\right)_{0} \\
+i \operatorname{Res}(0,0)_{0} O_{\alpha \beta}\left(0, \vec{q}, 0, i m_{0}, i m_{0}\right)_{1}
\end{aligned}
$$

where the subscripts 0,1 indicate the order in $1 / N$ to which the quantity has to be computed.

The $1 / N$ corrections to the $z$ self-energy are shown in Fig. 2 (we drop $\lambda$ tadpole diagrams). Of these only the last one couples to the impurity and, therefore, breaks translational invariance. So, letting,

$$
\begin{gathered}
D\left(\vec{k}, \vec{k}^{\prime}, \omega\right)=D_{0}\left(\vec{k}, \vec{k}^{\prime}, \omega\right)-\sum_{\vec{p}, \vec{p}^{\prime}} D_{0}(\vec{k}, \vec{p}, \omega) \Sigma\left(\vec{p}, \vec{p}^{\prime}, \omega\right) D\left(\vec{p}^{\prime}, \vec{k}^{\prime}, \omega\right) \\
\Sigma\left(\vec{k}, \vec{k}^{\prime}, \omega\right) \stackrel{\vec{k} \neq \vec{k}^{\prime}}{=} \frac{1}{L^{2}} 2 i \omega \mathcal{D}_{\tau \tau}\left(\vec{k}-\vec{k}^{\prime}, 0\right)+O\left(1 / N^{2}\right)
\end{gathered}
$$

and

$$
D\left(\vec{k}, \overrightarrow{k^{\prime}}, \omega\right) \stackrel{\vec{k} \neq \vec{k}^{\prime}}{=}-\frac{1}{L^{2}} 2 i \omega \mathcal{D}_{\tau \tau}\left(\vec{k}-\vec{k}^{\prime}, 0\right) \frac{1}{\omega^{2}+\vec{k}^{2}+m_{0}^{2}} \frac{1}{\omega^{2}+\vec{k}^{\prime 2}+m_{0}^{2}}+O\left(1 / N^{2}\right)
$$

So the residue,

$$
i \operatorname{Res}(0,-\vec{q})=i \operatorname{Res}(\vec{q}, 0) \stackrel{\vec{q} \neq 0}{=} \frac{1}{L^{2}} \frac{1}{\vec{q}^{2}} \mathcal{D}_{\tau \tau}(\vec{q}, 0)+O\left(1 / N^{2}\right)
$$

Note that at this order renormalization of the location of the pole $\omega=i m \stackrel{N=\infty}{=} i m_{0}$ can be neglected.

The $1 / N$ corrections to the insertion of $Q^{a}$ into the $z$ propagator are shown in Fig. 3 .

Again, only the last one of these couples to the impurity and breaks translational invariance, so,

$$
Q_{\alpha \beta}^{a}\left(\vec{p}, \vec{q}, \vec{p}^{\prime}, \omega, \omega^{\prime}\right) \stackrel{\vec{q} \neq \vec{p}^{\prime}-\vec{p}}{=}-2 \frac{1}{L^{2}} \mathcal{D}_{\tau \tau}\left(\vec{q}+\vec{p}-\vec{p}^{\prime}, 0\right) T_{\alpha \beta}^{a}+O\left(1 / N^{2}\right)
$$

Combining (3.42),(3.46),(3.47),

$$
\left\langle\alpha\left|Q^{a}(\vec{q})\right| \beta\right\rangle=-\left(\delta_{\vec{q}, 0}+\left(1-\delta_{\vec{q}, 0}\right) \frac{1}{\theta L}\left(1+\frac{4 m_{0}^{2}}{\vec{q}^{2}}\right) \mathcal{D}_{\tau \tau}(\vec{q}, 0)\right) T_{\alpha \beta}^{a}+O\left(1 / N^{2}\right)
$$



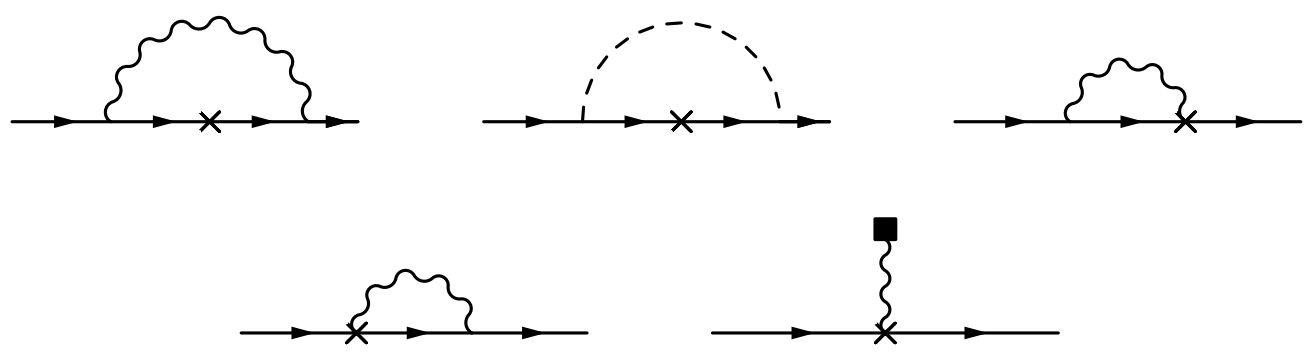

FIG. 3: $1 / N$ corrections to the insertion of $Q^{a}$ into the $z$ propagator.

The calculation of $1 / N$ corrections to result (3.40) for impurity induced staggered magnetization $n^{a}(x)$ proceed in the same fashion. The corrections to insertion of $n^{a}(x)$ into the $z$ propagator are given by the first two diagrams in Fig. 3 (except now the cross stands for $n^{a}$ insertion). None of these break translational invariance (as the last diagram in Fig. 3 is present only for $Q^{a}$, but not for $n^{a}$ ). Therefore,

$$
\left\langle\alpha\left|n^{a}(\vec{q})\right| \beta\right\rangle=\frac{1}{2 m_{0}}\left(\delta_{\vec{q}, 0}(1+O(1 / N))+\left(1-\delta_{\vec{q}, 0}\right) \frac{1}{L^{2}} \frac{4 m_{0}}{\vec{q}^{2}} \mathcal{D}_{\tau \tau}(\vec{q}, 0)\right) T_{\alpha \beta}^{a}+O\left(1 / N^{2}\right)
$$

Note again that in the case of $\left\langle\alpha\left|n^{a}(\vec{q})\right| \beta\right\rangle$ we have computed the $1 / N$ corrections only to $\vec{q} \neq 0$. Unlike the case of uniform magnetization, here the $N=\infty$ result for $\left\langle\alpha\left|n^{a}(\vec{q}=0)\right| \beta\right\rangle$ is expected to receive corrections.

Thus, the scaling functions,

$$
\begin{aligned}
& \Phi_{Q}(\vec{x} / L)=-1-\frac{1}{\theta L} \sum_{\vec{q} \neq 0}\left(1+\frac{4 m_{0}^{2}}{\vec{q}^{2}}\right) \mathcal{D}_{\tau \tau}(\vec{q}, 0) e^{i \vec{q} \vec{x}}+O\left(1 / N^{2}\right) \\
& \Phi_{n}(\vec{x} / L)=\frac{1}{2 \theta}+c_{1}+\frac{1}{L^{3}} \sum_{\vec{q} \neq 0} \frac{2}{\vec{q}^{2}} \mathcal{D}_{\tau \tau}(\vec{q}, 0) e^{i \vec{q} \vec{x}}+O\left(1 / N^{2}\right)
\end{aligned}
$$

where $c_{1}$ is an $\vec{x}$-independent constant of order $1 / N\left(c_{1}\right.$ should be also independent of $\Lambda$; we have not verified this fact as we did not compute the $1 / N$ corrections to $\left.\left\langle\alpha\left|n^{a}(\vec{q}=0)\right| \beta\right\rangle\right)$. We may write,

$$
\begin{aligned}
\Phi_{Q}(\vec{r}) & =-\left(1+\frac{1}{N} f_{Q}(\vec{r})\right)+O\left(1 / N^{2}\right) \\
\Phi_{n}(\vec{r}) & =\frac{1}{2 \theta}\left(1+2 c_{1} \theta+\frac{1}{N} f_{n}(\vec{r})\right)+O\left(1 / N^{2}\right)
\end{aligned}
$$

We have evaluated the functions $f_{Q}, f_{n}$ numerically and plotted them along the diagonal of our spatial torus in Fig. 4.

Now, we would like to find the $\vec{q} \rightarrow \infty, \vec{x} \rightarrow 0$ asymptotes of (3.50), (3.51). For this 

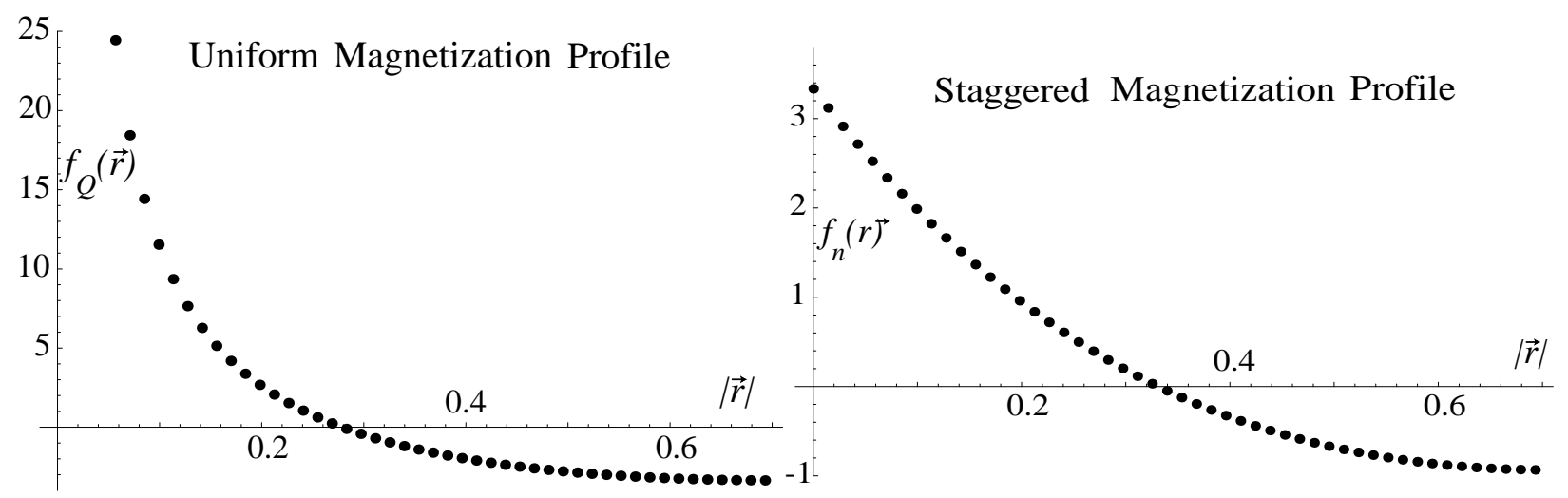

FIG. 4: Uniform (a) and Staggered (b) magnetization distribution functions $f_{Q}(\vec{r}), f_{n}(\vec{r})$ plotted along the torus diagonal.

purpose, we may replace the finite box propagator $\mathcal{D}_{\tau \tau}(q)$ by the infinite box propagator,

$$
\mathcal{D}_{\tau \tau}(\vec{q}, 0) \stackrel{\vec{q} \rightarrow \infty}{\rightarrow} \frac{1}{N A} \frac{1}{|\vec{q}|}
$$

Writing, $\Phi_{Q, n}(\vec{x} / L)=\frac{1}{L^{2}} \sum_{\vec{q}} \Phi_{Q, n}(\vec{q}) e^{i \vec{q} \vec{x}}$

$$
\begin{aligned}
& \Phi_{Q}(\vec{q}) \stackrel{\vec{q} \rightarrow \infty}{\rightarrow}-\frac{1}{N A \theta} \frac{L}{|\vec{q}|}+O\left(1 / N^{2}\right) \\
& \Phi_{n}(\vec{q}) \stackrel{\vec{q} \rightarrow \infty}{\rightarrow} \frac{2}{N A} \frac{1}{L|\vec{q}|^{3}}+O\left(1 / N^{2}\right)
\end{aligned}
$$

Fourier transforming,

$$
\begin{aligned}
& \Phi_{Q}(\vec{r}) \stackrel{|\vec{r}| \rightarrow 0}{\rightarrow}-\frac{1}{2 \pi \theta N A} \frac{1}{|\vec{r}|}+O\left(1 / N^{2}\right) \\
& \Phi_{n}(\vec{r}) \stackrel{|\vec{r}| \rightarrow 0}{\rightarrow} \frac{1}{2 \theta}+c_{2}+O\left(1 / N^{2}\right)
\end{aligned}
$$

where $c_{2}$ is a constant of order $1 / N$.

Thus, we conclude that,

$$
\Delta_{\mathrm{imp}}^{Q}=-1+O(1 / N) \quad \Delta_{\mathrm{imp}}^{n}=O\left(1 / N^{2}\right)
$$

which is consistent with the relation between impurity exponents (3.11). Note that the present calculation shows that $\Delta_{\text {imp }}^{n}$ is zero to order $1 / N$. We shall verify this fact in a different way in Section III B, and compute $\Delta_{\text {imp }}^{n}$ to order $1 / N^{2}$.

Moreover, the ratio,

$$
\frac{c_{Q}}{c_{n}}=-\frac{1}{\pi N A}+O\left(1 / N^{2}\right)
$$


is independent of regularization at this order in $N$.

\section{B. Néel Phase}

In this section, we compute the uniform and staggered magnetization in the presence of an impurity of charge $Q$ in the symmetry broken phase, $g<g_{c}$. We work in infinite volume. We develop the $1 / N$ expansion around the symmetry broken vacuum,

$$
\left\langle z_{1}\right\rangle=\frac{1}{\sqrt{2}} v
$$

Note that in general $v$ is not a gauge invariant quantity. However, this fact does not manifest itself at the order at which we are working. To leading order in $N$,

$$
\frac{1}{2} v^{2}=\frac{1}{g}-\frac{1}{g_{c}}
$$

Note that $v^{2} \sim O(N)$. Moreover, we take $Q \sim O(1)$ in $N$.

We now must quantize our theory around the symmetry broken state. We write,

$$
z_{1}=\frac{1}{\sqrt{2}}(h+v+i \phi), \quad z_{\alpha}=\pi_{\alpha}, \alpha=2 . . N
$$

We work in the so-called $R_{\xi}$ gauge, in which the mixing between the goldstone $\phi$ and the photon $A_{\mu}$ is absent, at the expense of introducing a ghost field $c$. In what follows, we have eliminated the mixing only to leading order in $1 / N$. This is achieved by using the gauge-fixing condition,

$$
\partial_{\mu} A_{\mu}=\xi v K^{-1} \phi+w
$$

where the action for the auxillary field $w$, which appears in the Fadeev-Poppov formalism, is

$$
\mathcal{S}_{w}=\frac{1}{2 \xi} \int d x d y w(x) K(x-y) w(y)
$$

Here, $K(x-y)$ is the photon polarization function given by Eq. (3.31). Similarly, in what follows $\Pi(x-y)$ is the $\lambda$ self energy given by Eq. (3.33). 
At the end of the day, the action one obtains is,

$$
\begin{aligned}
\mathcal{S}_{\xi} & =\frac{1}{2} \int d x d y A_{\mu}(x)\left(K_{\mu \nu}(x-y)-\frac{1}{\xi} \partial_{\mu} \partial_{\nu} K(x-y)+\delta_{\mu \nu} v^{2}\right) A_{\nu} \\
& +\frac{1}{2} \int d x d y \phi(x)\left(-\partial^{2} \delta(x-y)+\xi v^{2} K^{-1}(x-y)\right) \phi(y)+\frac{1}{2} \int d x d y \lambda(x) \Pi(x-y) \lambda(y) \\
& +\int d x d y\left(\bar{c}\left(-\partial^{2} \delta(x-y)+\xi v^{2} K^{-1}(x-y)\right) c(y)+\xi v \bar{c}(x) K^{-1}(x-y) h(y) c(y)\right) \\
& +\int d x\left(\left|D_{\mu} \pi\right|^{2}+\frac{1}{2}\left(\partial_{\mu} h\right)^{2}+i v \lambda h+\left(\phi \partial_{\mu} h-\partial_{\mu} \phi h\right) A_{\mu}+\left(v h+\frac{1}{2} h^{2}+\frac{1}{2} \phi^{2}\right) A_{\mu}^{2}\right) \\
& +\int d x\left(i \lambda|\pi|^{2}+\frac{1}{2} i \lambda\left(\phi^{2}+h^{2}\right)\right)
\end{aligned}
$$

As usual, we avoid double counting by dropping any diagrams, which are already included in the dynamically generated $N=\infty$ self-energies for $A_{\mu}, \lambda$ etc. The propogators for our fields are shown in Fig. 5. Note that in the Néel phase, we get mixing between the $\lambda$ and $h$ fields.

$$
\begin{aligned}
& A_{\mu} \sim \sim \sim \sim A_{\nu} \quad \mathcal{D}_{\mu \nu}(p)=\frac{1}{p^{2} K(p)+v^{2}}\left(\delta_{\mu \nu}+\frac{(\xi-1) K(p) p_{\mu} p_{\nu}}{p^{2} K(p)+\xi v^{2}}\right) \\
& \phi \text { WWWWWWWW } \phi \quad D_{\phi}(p)=\frac{1}{p^{2}+\xi v^{2} K^{-1}(p)} \\
& \bar{c} \ldots \ldots \ldots \cdots \cdots \cdots \cdots+D_{c}(p)=\frac{1}{p^{2}+\xi v^{2} K^{-1}(p)} \\
& \pi_{\alpha}^{*} \longrightarrow \pi_{\beta} \quad D_{\pi_{\alpha \beta}}(p)=\frac{\delta_{\alpha \beta}}{p^{2}} \\
& h \longrightarrow h \quad D_{h}(p)=\frac{\Pi(p)}{p^{2} \Pi(p)+v^{2}} \\
& \lambda---------\lambda \quad D_{\lambda}(p)=\frac{p^{2}}{p^{2} \Pi(p)+v^{2}} \\
& h----\lambda \quad D_{h \lambda}(p)=\frac{-i v}{p^{2} \Pi(p)+v^{2}}
\end{aligned}
$$

FIG. 5: Propagators in the Néel phase.

Now, having set up the perturbation theory, we wish to compute, $\left\langle Q^{a}(\vec{x})\right\rangle,\left\langle n^{a}(\vec{x})\right\rangle$. Utilizing the pattern of spontaneous symmetry breaking, $U(N) \rightarrow U(N-1)$ (here we look 
FIG. 6: Leading contribution to uniform magnetization in the symmetry broken phase.

only at global symmetry), one can show that,

$$
\left\langle n^{a}\right\rangle=T_{11}^{a}\left\langle n^{0}\right\rangle
$$

where $n^{0}=z^{\dagger} T^{0} z$ and $T^{0}$ is any generator of $\mathrm{SU}(N)$ with $T_{11}^{0}=1$. Similarly for $Q^{a}$. For definiteness, we may choose $T_{11}^{0}=1, T_{1 \alpha}^{0}=T_{\alpha 1}^{0}=0, T_{\alpha \beta}^{0}=-\frac{1}{N-1} \delta_{\alpha \beta}, \alpha, \beta=2 \ldots N$.

Let's start with computing the uniform magnetization.

$$
Q^{0}=\frac{N}{N-1} j_{\tau}^{1}-\frac{1}{N-1} j_{\tau}
$$

where

$$
j_{\tau}^{1}=z_{1}^{\dagger} D_{\tau} z_{1}-\left(D_{\tau} z_{1}\right)^{\dagger} z_{1}
$$

and $j_{\tau}$ is the $U(1)$ charge density discussed in the appendix, see Eq. (A1). By equation of motion (A4),

$$
\left\langle j_{\tau}(\vec{x})\right\rangle=-J_{\tau}^{\text {ext }}(\vec{x})=-Q \delta^{2}(\vec{x})
$$

So, it remains to compute $\left\langle j_{\tau}^{1}(\vec{x})\right\rangle$. Expanding $j_{\tau}^{1}$ in terms of $\phi, h$ and $A_{\mu}$,

$$
j_{\tau}^{1}=-i v^{2} A_{\tau}+i v\left(\partial_{\tau} \phi-2 A_{\tau} h\right)+i\left(h \partial_{\tau} \phi-\phi \partial_{\tau} h-A_{\tau}\left(h^{2}+\phi^{2}\right)\right)
$$

In the $1 / N$ expansion the leading contribution to $\left\langle j_{\tau}^{1}\right\rangle$ is of $O(1)$ and comes from the first term on the r.h.s of (3.71), see Fig. 6.

$$
\left\langle j_{\tau}^{1}(\vec{p})\right\rangle=-Q v^{2} \mathcal{D}_{\tau \tau}(\vec{p}, 0)=-Q v^{2} \frac{1}{\vec{p}^{2} K(\vec{p})+v^{2}}
$$

Thus, $\left\langle Q^{0}(\vec{p})\right\rangle=\left\langle j_{\tau}^{1}(\vec{p})\right\rangle+O(1 / N)$. Fourier transforming,

$$
\left\langle Q^{0}(\vec{x})\right\rangle=-\frac{Q v^{2}}{2 \pi N A} \frac{1}{|\vec{x}|}+\frac{Q v^{4}}{4 N^{2} A^{2}}\left(\boldsymbol{H}_{0}\left(\frac{v^{2}|\vec{x}|}{N A}\right)-Y_{0}\left(\frac{v^{2}|\vec{x}|}{N A}\right)\right)+O(1 / N),
$$

where $\boldsymbol{H}_{0}$ is the Struve function and $Y_{0}$ is the Bessel function. Taking the short and long distance asymptotes,

$$
\begin{gathered}
\left\langle Q^{0}(\vec{x})\right\rangle \stackrel{\vec{x} \rightarrow 0}{\rightarrow}-\frac{Q v^{2}}{2 \pi N A} \frac{1}{|\vec{x}|} \\
\left\langle Q^{0}(\vec{x})\right\rangle \stackrel{\vec{x} \rightarrow \infty}{\rightarrow}-\frac{Q N A}{2 \pi v^{2}} \frac{1}{|\vec{x}|^{3}}
\end{gathered}
$$




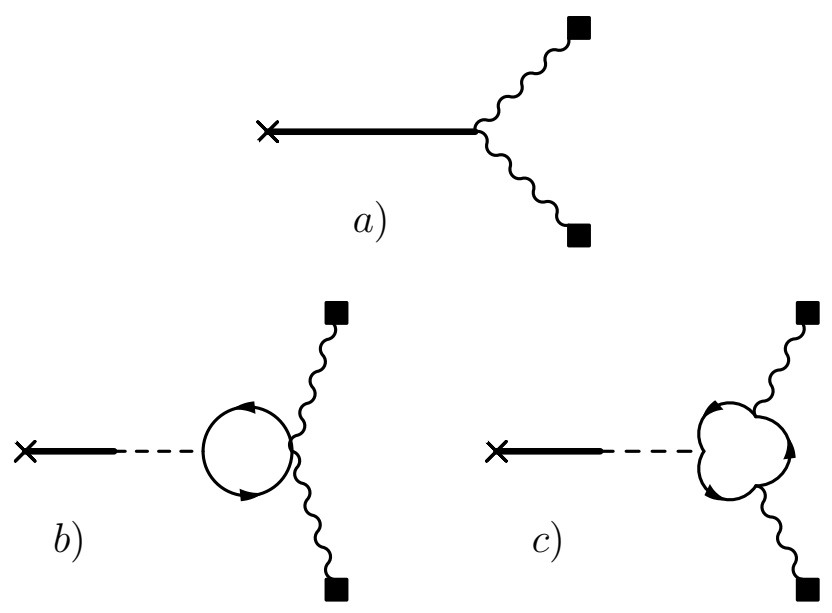

FIG. 7: Leading $\vec{x}$-dependent contribution to staggered magnetization in the symmetry broken phase.

The long distance decay is a consequence of the Goldstone physics of the spin waves, and the $1 /|\vec{x}|^{3}$ decay is expected to be exact. At short distances, we have the physics of the critical point, and the exponent will have corrections at higher order. From the present result we can conclude that the impurity exponent

$$
\Delta_{\mathrm{imp}}^{Q}=-1+O(1 / N)
$$

which is consistent with the result obtained at the critical point (3.59).

Now, let's discuss the staggered magnetization,

$$
n^{0}=\frac{N}{N-1} z_{1}^{\dagger} z_{1}-\frac{1}{N-1} z^{\dagger} z
$$

By equations of motion,

$$
z^{\dagger} z=\frac{1}{g}
$$

thus,

$$
n^{0}=\frac{N}{N-1} z_{1}^{\dagger} z_{1}-\frac{1}{(N-1) g}
$$

and

$$
z_{1}^{\dagger} z_{1}=\frac{1}{2} v^{2}+v h+\frac{1}{2}\left(h^{2}+\phi^{2}\right)
$$

Thus, at leading order, $\left\langle z_{1}^{\dagger} z_{1}(\vec{x})\right\rangle=\frac{1}{2} v^{2}$, and

$$
\left\langle n^{0}(\vec{x})\right\rangle=\frac{1}{2} v^{2}+O(1)
$$

Moreover, the $\vec{x}$-dependent corrections to $\left\langle n^{0}(\vec{x})\right\rangle$ come only at $O(1 / N)$, with diagrams of Fig. 7 (the part of $n^{0}$ which contributes at this order, denoted by $\times$, is $v h$ ). 

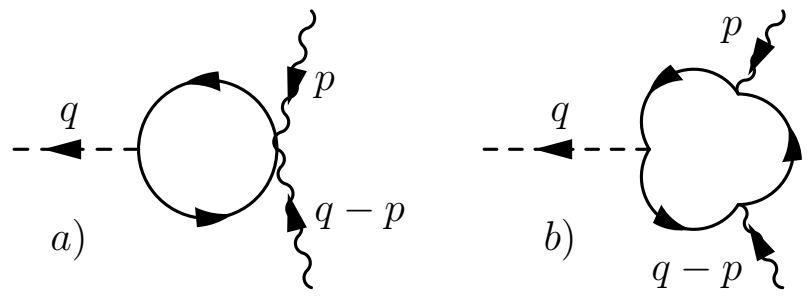

FIG. 8: Leading contribution to the three point vertex of $A_{\mu}, A_{\nu}$ and $\lambda$ fields, $\Gamma^{\mu \nu}(q, p, q-p)$.

We will discuss the diagrams in Fig. 7 shortly. For now, we can conclude that,

$$
\Delta_{\text {imp }}^{n}=O\left(1 / N^{2}\right)
$$

in agreement with the result (3.59) obtained at the critical point. Moreover, we can now compute the ratio,

$$
\frac{c_{Q}}{c_{n}}=-\frac{Q}{\pi N A}+O\left(1 / N^{2}\right)
$$

which exactly agrees with the result obtained at the critical point (3.60) for $Q=1$. Notice, that this is a highly nontrivial check of the OPE (3.8) as $\left\langle Q^{a}\right\rangle,\left\langle n^{a}\right\rangle$ depend on $v$ in the Néel phase and on $L$ at the critical point. Nevertheless, all the dependence on the IR scale cancels out in the ratio $c_{Q} / c_{n}$, which is constant throughout the scaling regime.

Coming back to the diagrams in Fig. 7,

$$
\left\langle n^{0}(\vec{q})\right\rangle \stackrel{\vec{q} \neq 0}{=} Q^{2} v^{2} D_{h}(\vec{q}, 0) \int \frac{d^{D-1} p}{(2 \pi)^{D-1}}\left(1+\frac{i}{2} \Pi^{-1}(\vec{q}, 0) \Gamma^{\tau \tau}(\vec{q}, 0, \vec{p}, 0, \vec{q}-\vec{p}, 0)\right) \mathcal{D}_{\tau \tau}(\vec{p}, 0) \mathcal{D}_{\tau \tau}(\vec{q}-\vec{p}, 0)
$$

We keep the space-time dimension $D$ arbitrary in what follows, as we wish to compare our result for $\Delta_{\text {imp }}^{n}$ obtained in the $1 / N$ expansion, with the result obtained using $\epsilon$ expansion ${ }^{30}$. Here, $\Gamma^{\mu \nu}(q, p, q-p)$ is the lowest order contribution to the $A_{\mu}, A_{\nu}, \lambda$ vertex, given by the sum of the loops in Fig. 8. The diagram in Fig. 8 a) is given by,

$$
\Gamma_{1}^{\mu \nu}(q, p, q-p)=2 i \delta_{\mu \nu} \Pi(q)
$$

Thus, diagrams in Fig. $7 \mathrm{a}$ ) and b) cancel (by the way, these diagrams are individually UV divergent for $D \leq 3)$. So, calling the diagram in Fig. 8 b), $\Gamma_{2}^{\mu \nu}(q, p, q-p)$,

$$
\left\langle n^{0}(\vec{q})\right\rangle \stackrel{\vec{q} \neq 0}{=} Q^{2} v^{2} D_{h}(\vec{q}, 0) \int \frac{d^{D-1} p}{(2 \pi)^{D-1}} \frac{i}{2} \Pi^{-1}(\vec{q}, 0) \Gamma_{2}^{\tau \tau}(\vec{q}, 0, \vec{p}, 0, \vec{q}-\vec{p}, 0) \mathcal{D}_{\tau \tau}(\vec{p}, 0) \mathcal{D}_{\tau \tau}(\vec{q}-\vec{p}, 0)
$$


Evaluating $\Gamma_{2}^{\mu \nu}$

$$
\begin{aligned}
\Gamma_{2}^{\mu \nu}(q, p, q-p)= & -2 i N \int \frac{d^{D} l}{(2 \pi)^{D}} \frac{(2 l-p)_{\mu}(2 l-p-q)_{\nu}}{l^{2}(l-p)^{2}(l-q)^{2}} \\
= & -\frac{4 i N \Gamma(2-D / 2)}{(4 \pi)^{D / 2}} \int d x_{1} d x_{2} d x_{3} \delta\left(1-x_{1}-x_{2}-x_{3}\right)\left(\Delta^{2}\right)^{D / 2-2} \\
& \left(\delta_{\mu \nu}+\frac{(4-D)\left(2 x_{1} q+\left(2 x_{2}-1\right) p\right)_{\mu}\left(\left(2 x_{1}-1\right) q+\left(2 x_{2}-1\right) p\right)_{\nu}}{4 \Delta^{2}}\right)
\end{aligned}
$$

where,

$$
\Delta^{2}=x_{1}\left(1-x_{1}\right) q^{2}+x_{2}\left(1-x_{2}\right) p^{2}-2 x_{1} x_{2} p \cdot q
$$

We are interested only in $\Gamma_{2}^{\tau \tau}$, with $p^{0}=q^{0}=0$. Thus,

$$
\Gamma_{2}^{\tau \tau}(\vec{q}, 0, \vec{p}, 0, \vec{q}-\vec{p}, 0)=-\frac{4 i N \Gamma(2-D / 2)}{(4 \pi)^{D / 2}} \int d x_{1} d x_{2} d x_{3} \delta\left(1-x_{1}-x_{2}-x_{3}\right)\left(\Delta^{2}\right)^{D / 2-2}
$$

For $|\vec{p}| \gg|\vec{q}|, \Gamma_{2}^{\tau \tau}(\vec{q}, 0, \vec{p}, 0, \vec{q}-\vec{p}, 0) \sim|\vec{p}|^{D-4}$, so for $\vec{p} \rightarrow \infty$ the integrand in Eq. (3.86) behaves as $|\vec{p}|^{-D}$ and the integral is UV convergent.

We now attempt to understand the behaviour of (3.86) for $\vec{q} \rightarrow \infty$, from which we should be able to extract the impurity anomalous dimension $\Delta_{\mathrm{imp}}^{n}$. For this purpose, we may set $v=0$ in the propagators $D_{h}(\vec{q}, 0), \mathcal{D}_{\tau \tau}(\vec{p}, 0), \mathcal{D}_{\tau \tau}(\vec{q}-\vec{p}, 0)$ (this does not introduce any IR divergences).

$$
\left\langle n^{0}(\vec{q})\right\rangle \stackrel{\vec{q} \rightarrow \infty}{=} \frac{Q^{2} v^{2}}{N^{3} A^{2} B}|\vec{q}|^{2-D} \int \frac{d^{D-1} p}{(2 \pi)^{D-1}} \frac{i}{2} \Gamma_{2}^{\tau \tau}(\vec{q}, 0, \vec{p}, 0, \vec{q}-\vec{p}, 0) \frac{1}{|\vec{p}|^{D-2}} \frac{1}{|\vec{p}-\vec{q}|^{D-2}}
$$

Let us first discuss the limit $D=4-\epsilon, \epsilon \rightarrow 0$. In this regime, to leading order in $\epsilon$,

$$
\Gamma_{2}^{\tau \tau}(\vec{q}, 0, \vec{p}, 0, \vec{q}-\vec{p}, 0)=-2 i N \frac{1}{(4 \pi)^{2}} \Gamma(2-D / 2)
$$

and,

$$
\left\langle n^{0}(\vec{q})\right\rangle \stackrel{\vec{q} \rightarrow \infty}{=} \frac{72 \pi^{4} \epsilon^{2} Q^{2} v^{2}}{N^{2}} \frac{1}{|\vec{q}|^{3}}
$$

Fourier transforming,

$$
\left\langle n^{0}(\vec{x})\right\rangle \stackrel{\vec{x} \rightarrow 0}{=} \frac{1}{2} v^{2}+c_{3}-\frac{36 \pi^{2} \epsilon^{2} Q^{2}}{N^{2}} v^{2} \log (v|\vec{x}|)+c_{4}+O\left(1 / N^{2}\right)
$$

where $c_{3}, c_{4}$ do not depend on $\vec{x}$ and are of order 1 and $1 / N$ respectively. Thus, to leading 
order in $1 / N, \epsilon$,

$$
\Delta_{\text {imp }}^{n}=-\frac{72 \pi^{2} Q^{2} \epsilon^{2}}{N^{2}}
$$

in agreement with the calculations of Ref. 30, where the impurity exponents were obtained by performing the impurity operator renormalizations as summarized in Section III A $1 .^{3}$

For arbitrary $D, \Delta_{\text {imp }}^{n}$ is difficult to calculate analytically, as $\Gamma^{\tau \tau}$ is no longer a constant. However, combining Eqs. (3.86), (3.89) and introducing a new set of Feynman parameters,

$$
\left\langle n^{0}(\vec{q})\right\rangle \stackrel{\vec{q} \rightarrow \infty}{=} \frac{Q^{2} v^{2}}{N^{2}} \frac{1}{|\vec{q}|^{D-1}} f(D)
$$

where the numerical constant $f(D)$ is given by,

$$
\begin{aligned}
& f(D)=\frac{1}{A^{2} B(4 \pi)^{D-1} \Gamma(D / 2-1)^{2}} \int_{0}^{1} d x_{1} \int_{0}^{1-x_{1}} d x_{2} \int_{0}^{1} d y_{1} \int_{0}^{1-y_{1}} d y_{2} \\
& x_{2}^{(D-3) / 2}\left(1-x_{2}\right)^{D / 2-1} y_{1}^{1-D / 2} y_{2}^{D / 2-2}\left(1-y_{1}-y_{2}\right)^{D / 2-2} \\
& \left(x_{2}\left(1-x_{2}\right)^{2} y_{2}\left(1-y_{2}\right)+x_{1} y_{1}\left(\left(1-x_{1}\right)\left(1-x_{2}\right)-2 y_{2} x_{2}\left(1-x_{2}\right)-y_{1} x_{1} x_{2}\right)\right)^{-\frac{1}{2}}
\end{aligned}
$$

Consequently,

$$
\left\langle n^{0}(\vec{x})\right\rangle \stackrel{\vec{x} \rightarrow 0}{=} \frac{1}{2} v^{2}+c_{3}-\frac{2}{(4 \pi)^{(D-1) / 2} \Gamma((D-1) / 2)} f(D) \frac{Q^{2}}{N^{2}} v^{2} \log \left(v^{2 /(D-2)}|\vec{x}|\right)+c_{4}+O\left(1 / N^{2}\right)
$$

and

$$
\Delta_{\text {imp }}^{n}=-\frac{4}{(4 \pi)^{(D-1) / 2} \Gamma((D-1) / 2)} f(D) \frac{Q^{2}}{N^{2}}+O\left(1 / N^{3}\right)
$$

Evaluating $f(D)$ numerically for $D=3$,

$$
\Delta_{\text {imp }}^{n} \approx-25.9 \frac{Q^{2}}{N^{2}}+O\left(1 / N^{3}\right)
$$

We note that we have separately verified the result (3.98) by performing the impurity OPE program as summarized in Section III A 1.

\footnotetext{
${ }^{3}$ Note that in the $\epsilon$ expansion of Ref. 30 only the analogue of the diagram in Fig. 7 a) appears, while the diagrams in Figs. 7 b), c) do not appear at leading order in $\epsilon$, as they are higher order in coupling constant. Nevertheless in the $1 / N$ expansion, we saw that the answer comes entirely from the diagram in Fig. $7 \mathrm{c}$ ), with diagrams in Fig. 7 a) and Fig. $7 \mathrm{~b}$ ) canceling for all $D$. The reason is the following: in the $1 / N$ expansion all diagrams in Fig. 7 are individually of same order in $\epsilon$. Moreover, to leading order in $\epsilon$, the diagrams b) and c) cancel, so a) $=-$ b) $\stackrel{\epsilon \rightarrow 0}{=}$ c). In the $\epsilon$ expansion, this fact is foreseen in advance: the $1 / \epsilon$ pole must cancel between diagrams b) and c) (the 4-point diagram with two photons and two scalars is not divergent). Thus, we can obtain the answer to leading order in $\epsilon$ either from a) alone or from c) alone.
} 


\section{CONCLUSIONS}

A recent numerical study ${ }^{18}$ examined the spin distribution in the vicinity of a nonmagnetic impurity in a double-layer, $S=1 / 2$ square lattice antiferromagnet at its quantum critical point. The ground state of the system has total spin $S=1 / 2$, and the spin distribution of this $S=1 / 2$ was found to be extended across the entire system. Universal scaling forms (Eqs. (1.3) and (1.5)) for the uniform and staggered spin distributions were postulated $^{18}$, and found to be in excellent agreement with the numerical results.

This paper has presented the field-theoretic foundation of the above results. Using the soft-spin O(3) LGW field theory in Eq. (2.2), we found that the universal scaling forms in Eqs. (1.3) and (1.5) were indeed obeyed in an expansion in $(3-d)$ (where $d$ is the spatial dimensionality), and explicit results for the universal scaling functions appear in Eq. (2.13).

Next, we examined a similar non-magnetic impurity in $S=1 / 2$ antiferromagnets which have a single $S=1 / 2$ spin per unit cell. Such antiferromagnets can display a deconfined quantum phase transition ${ }^{24,25}$ between Néel and valence bond solid (VBS) states. An explicit example of such a transition was found recently in Ref. 23. We expect that such studies will be extended to include non-magnetic impurities in the future, and so have provided our theoretical predictions here. The field theory for this situation in $\mathcal{S}_{b}^{z}+\mathcal{S}_{\text {imp }}^{z}$ in Eqs. $(1.7,1.8)$. It describes the dynamics of a $\mathrm{SU}(N)$ spinor field, $z_{\alpha}$ (the spinon), and we obtained its critical properties in a $1 / N$ expansion. Projecting onto the total spin $S=1 / 2$ sector of this theory (which contains the ground state in the presence of the impurity) was not straightforward here, and we achieved this by the relation Eq. (1.9). Our results obey scaling forms which appear in Section III A 1. The scaling functions are in Eqs. (3.50) and (3.51), and are plotted in Fig. 4. The boundary spin exponent for the deconfined critical point appears in Eqs. (3.98), (3.99). We also obtained substantial evidence for the structure of the operator product expansion near the impurity, and the fact that the staggered and uniform magnetizations flow to the same impurity spin operator.

Our study of the deconfined case has so far only examined the uniform and staggered spin magnetizations near the non-magnetic impurity. We have not provided here a description of the structure of the VBS order near the impurity. This will be presented in a forthcoming paper.

\section{Acknowledgments}

We are grateful to A. Sandvik for valuable discussions. This research was supported by the NSF grant DMR-0537077.

\section{APPENDIX A: $U(1)$ CHARGE DENSITY}

Throughout the paper we have concentrated on computing matrix elements of uniform and staggered magnetization $Q^{a}(x), n^{a}(x)$. However, for the deconfined critical point, it is 
also interesting to compute the charge density associated with the $U(1)$ local symmetry of the $\mathbb{C} \mathbb{P}^{N-1}$ model. This charge density is the zeroth component of the current,

$$
j_{\mu}(x)=z^{\dagger} D_{\mu} z-\left(D_{\mu} z\right)^{\dagger} z
$$

As we shall see this computation serves as an additional test of our procedure for projecting onto the single spinon state.

Consider the $\mathbb{C} \mathbb{P}^{N-1}$ model coupled to an external current,

$$
\mathcal{S}=\mathcal{S}_{b}^{z}+i \int d^{3} x A_{\mu} J_{\mu}^{\mathrm{ext}}
$$

As in the rest of the paper, we set $e^{2}=\infty$, so that the gauge field has no bare kinetic term. Then, by equations of motion,

$$
\begin{gathered}
0=\frac{\delta \mathcal{S}}{\delta A_{\mu}}=i\left(j^{\mu}+J_{\mu}\right) \\
j_{\mu}=-J_{\mu}^{\mathrm{ext}}
\end{gathered}
$$

Thus, the dynamical current completely screens (locally!) the external current. Eq. (A4) is an operator identity, and should, in particular, hold in the ground state of the system with a single impurity. Let's check this statement in the $1 / N$ expansion.

We start from Eq. (1.9), with $O(x)=j_{0}(x)$. We write,

$$
\left\langle\alpha\left|j_{0}(\vec{x})\right| \beta\right\rangle=\rho(\vec{x}) \delta_{\alpha \beta}
$$

with $\rho(\vec{x})=\frac{1}{L^{2}} \sum_{\vec{q}} \rho(\vec{q}) e^{i \vec{q} \vec{x}}$. The Wilson line term in Eq. (1.9) can be incorporated into the action as coupling to an external current, $J_{\mu}^{\text {ext }}(\vec{x}, \tau)=\delta_{\mu 0} \delta^{3}(\vec{x}) \theta(\mathcal{T} / 2-\tau) \theta(\tau+\mathcal{T} / 2)$. At leading order in $1 / N$ the numerator of Eq. (1.9) is given by diagrams shown in Fig. 9, while the denominator is given by the bare propagator $D(\vec{x}=0, \mathcal{T})$.

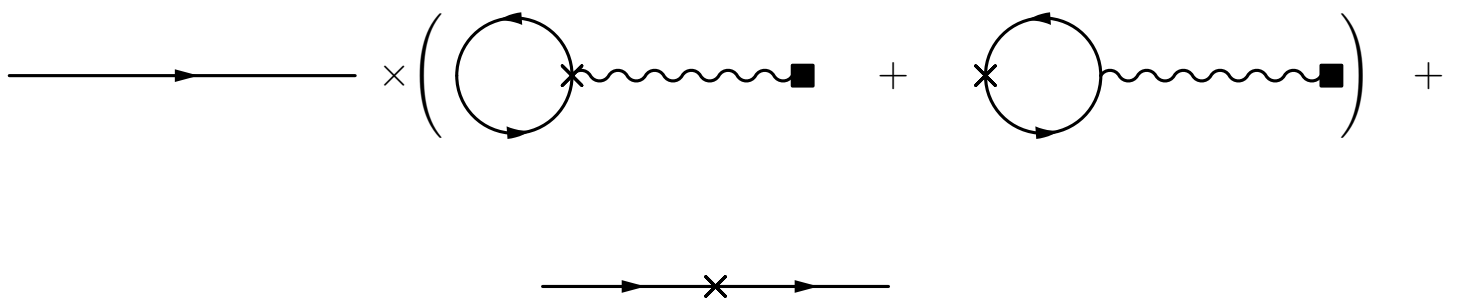

FIG. 9: Diagrams contributing to $U(1)$ charge induced

Thus, we can distinguish two contributions: the disconnected one, $\rho^{1}(\vec{q})$, coming from the first line in Fig. 9, and the connected one, $\rho^{2}(\vec{q})$, coming from the second line. We note 
that the scalar loops contributing to $\rho^{1}(\vec{q})$ are precisely the same as those contributing to the self-energy of $A_{\mu}$ field, thus,

$$
\rho^{1}(\vec{q})=-\int \frac{d q_{\tau}}{2 \pi} K_{0 \nu}\left(\vec{q}, q_{\tau}\right) \mathcal{D}_{\nu \lambda}\left(\vec{q}, q_{\tau}\right) J_{\lambda}^{\mathrm{ext}}\left(\vec{q}, q_{\tau}\right)
$$

Now,

$$
K_{\mu \nu}(q) \mathcal{D}_{\nu \lambda}(q)=\delta_{\mu \lambda}-\frac{q_{\mu} q_{\lambda}}{q^{2}}
$$

Thus,

$$
\rho^{1}(\vec{q})=-\int \frac{d q_{\tau}}{2 \pi} \frac{\vec{q}^{2}}{q_{\tau}^{2}+\vec{q}^{2}} J^{\mathrm{ext}}\left(\vec{q}, q_{\tau}\right)
$$

Noting, $J^{\text {ext }}\left(\vec{q}, q_{\tau}\right) \stackrel{\mathcal{T} \rightarrow \infty}{\rightarrow} 2 \pi \delta\left(q_{\tau}\right)$

$$
\rho^{1}(\vec{q})=-\left(1-\delta_{\vec{q}, 0}\right)
$$

For the $\vec{q}=0$ part, the order of the limits $\vec{q} \rightarrow 0, q_{\tau} \rightarrow 0$ is very important. In our finite system the $\vec{q}=0$ mode is isolated, and, moreover, in our present treatment the Wilson line is of finite length, so we must take the $\vec{q}=0$ limit first and then $q_{\tau} \rightarrow 0$. Hence, $\rho^{1}(\vec{q}=0)=0$. This is not surprising. In perturbation theory, we start with the vacuum which has charge 0 . Unless we manually project the system into a finite charge subspace (as we do in our treatment by acting on the vacuum with $z, z^{\dagger}$ operators), we will never be able to see global screening of charge. Since the diagrams contributing to $\rho^{1}(\vec{q})$ are disconnected from the external $z$ line, $\rho^{1}(\vec{q}=0)=0$.

Now, the connected contribution, simply gives the charge density of one spinon in the $\vec{k}=0$ state,

$$
\rho^{2}(\vec{q})=-\delta_{\vec{q}, 0}
$$

Putting the two contributions together,

$$
\begin{gathered}
\rho(\vec{q})=1 \\
\rho(\vec{x})=\delta^{2}(\vec{x})
\end{gathered}
$$

as expected by equations of motion (A4).

Thus, we have been able to check exact screening of external charge, which follows from equation of motion (A4), to leading order in $1 / N$. We see that local and global parts of the screening charge come from very different Feynman diagrams.

* Electronic address: mmetlits@fas.harvard.edu

$\dagger$ Electronic address: subir_sachdev@harvard.edu

1 K. Manabe, H. Ishimoto, N. Koide, Y. Sasago, and K. Uchinokura, Phys. Rev. B 58, R575 
(1998).

2 J. Bobroff, H. Alloul, W. A. MacFarlane, P. Mendels, N. Blanchard, G. Collin, and J.F. Marucco, Phys. Rev. Lett. 86, 4116 (2001).

3 S. Ouazi, J. Bobroff, H. Alloul, M. Le Tacon, N. Blanchard, G. Collin, M. H. Julien, M. Horvatić, and C. Berthier, Phys. Rev. Lett. 96, 127005 (2006).

4 A. Yazdani, B. A. Jones, C. P. Lutz, M. F. Crommie, and D. M. Eigler, Science 275, 1767 (1997).

5 S. H. Pan, E. W. Hudson, K. M. Lang, H. Eisaki, S. Uchida, J. C. Davis, Nature (London) 403, 746 (2000); E W. Hudson, K. M. Lang, V. Madhavan, S. H. Pan, H. Eisaki, S. Uchida, and J. C. Davis, Nature (London) 411, 920 (2001).

6 O. P. Vajk, P. K. Mang, M. Greven, P. M. Gehring, and J. W. Lynn, Science 295, 1691 (2002).

7 N. Nagaosa, Y. Hatsugai, and M. Imada, J. Phys. Soc. Jpn 58, 978 (1989).

8 A. W. Sandvik, E. Dagotto, and D. J. Scalapino, Phys. Rev. B 56, 11701 (1997).

9 S. Sachdev, C. Buragohain, and M. Vojta, Science 286, 2479 (1999)

10 M. Vojta, C. Buragohain, and S. Sachdev, Phys. Rev. B 61, 15152 (2000).

11 O. P. Sushkov, Phys. Rev. B 62, 12135 (2000).

12 A. L. Chernyshev, Y. C. Chen, and A. H. Castro Neto, Phys. Rev. B 65, 104407 (2002).

13 M. Troyer, Prog. Theor. Phys. Supplement 145, 326 (2002).

14 K. H. Höglund and A. W. Sandvik, Phys. Rev. Lett. 91, 077204 (2003).

15 S. Sachdev, and M. Vojta, Phys. Rev. B 68, 064419 (2003).

16 A. Lüscher and O. P. Sushkov, Phys. Rev. B 71, 064414 (2005).

17 A. V. Syromyatnikov and S. V. Maleyev, Phys. Rev. B 74, 184433 (2006).

18 K. H. Höglund, A. W. Sandvik, and S. Sachdev, Phys. Rev. Lett. 98, 087203 (2007).

19 K. H. Höglund and A. W. Sandvik, cond-mat/0701472.

20 V. N. Kotov, O. Sushkov, Z. Weihong, and J. Oitmaa, Phys. Rev. Lett. 80, 5790 (1998).

21 M. Matsumoto, C. Yasuda, S. Todo, and H. Takayama, Phys. Rev. B 65, 014407 (2002).

22 L. Wang, K. S. D. Beach, and A. W. Sandvik, Phys. Rev. B 73, 014431 (2006).

23 A. W. Sandvik, cond-mat/0611343.

24 T. Senthil, A. Vishwanath, L. Balents, S. Sachdev, and M. P. A. Fisher, Science 303, 1490 (2004)

25 T. Senthil, L. Balents, S. Sachdev, A. Vishwanath, and M. P. A. Fisher, Phys. Rev. B 70, 144407 (2004).

26 N. Read and S. Sachdev, Phys. Rev. Lett. 62, 1694 (1989); Phys. Rev. B 42, 4568 (1990).

27 A. DAdda, P. Di Vecchia, and M. Lüscher, Nucl. Phys. B 146, 63 (1978).

28 E. Witten, Nucl. Phys. B 149, 285 (1979).

29 O. I. Motrunich and A. Vishwanath, Phys. Rev. B 70, 075104 (2004).

30 A. Kolezhuk, S. Sachdev, R. R. Biswas, P. Chen, Phys. Rev. B 74, 165114 (2006).

31 R. K. Kaul, A. Kolezhuk, M. Levin, S. Sachdev, and T. Senthil, cond-mat/0702119.

32 S. Sachdev, Phys. Rev. B 55, 142 (1997).

33 V. Yu. Irkhin, A. A. Katanin, and M. I. Katsnelson, Phys. Rev. B 54, 11953 (1996). 